\title{
Analysis of the evolution of the practice of female genital mutilation/cutting in Burkina Faso
}

\author{
Nafissatou J. Diop \\ Population Council \\ Zakari Congo \\ Aina Ouedraogo \\ Alphosine Sawadogo \\ Lydia Saloucou
}

See next page for additional authors

Follow this and additional works at: https://knowledgecommons.popcouncil.org/departments_sbsr-rh

Part of the Demography, Population, and Ecology Commons, Family, Life Course, and Society Commons, Gender and Sexuality Commons, International Public Health Commons, Maternal and Child Health Commons, Sociology of Culture Commons, and the Women's Health Commons How does access to this work benefit you? Let us know!

\section{Recommended Citation}

Diop, Nafissatou J., Zakari Congo, Aina Ouedraogo, Alphosine Sawadogo, Lydia Saloucou, and Ida Tamini. 2008. "Analysis of the evolution of the practice of female genital mutilation/cutting in Burkina Faso," FRONTIERS Final Report. Washington, DC: Population Council. 


\section{Authors}

Nafissatou J. Diop, Zakari Congo, Aina Ouedraogo, Alphosine Sawadogo, Lydia Saloucou, and Ida Tamini 


\title{
Analysis of the Evolution of the Practice of Female Genital Mutilation/Cutting in Burkina Faso
}

\author{
Nafissatou J. Diop* \\ Zakari Congo* \\ Aïna Ouédraogo** \\ Alphonsine Sawadogo** \\ Lydia Saloucou* \\ Ida Tamini** \\ * Population Council \\ ** Comité National de Lutte contre la Pratique de l'Excision (CNLPE)
}

January 2008

This study was made possible by the generous support of the American people through the United States Agency for International Development (USAID) under the terms of Cooperative Agreement No. HRN-A00-98-00012-00 and Population Council Subaward No. AI05.01A and In-house project 5804 53090. The contents are the responsibility of the FRONTIERS Program and do not necessarily reflect the views of USAID or the United States Government.

1 Population Council

FRONTIIERS

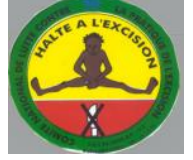

USAID 


\section{EXECUTIVE SUMMARY}

Virtually all ethnic groups in Burkina Faso practice FGM/C; the 2003 DHS indicated a national prevalence of 77 percent. The national campaign against FGM/C has been ongoing for nearly 20 years. Several international conventions have condemned FGM/C as a violation of the rights of human beings, the rights of children, and the right to health and to physical integrity. By signing these conventions, Burkina Faso has made the fight against FGM/C one of its priorities. The creation of a favourable environment has led to a diversity of action to encourage its abandonment, and so the situation in Burkina Faso can provide many lessons to others. It was one of the first countries to pioneer passing a law banning FGM/C and is probably the only African country to systematically enforce the law. This study was undertaken as a collaboration between the Population Council's Frontiers in reproductive health program (FRONTIERS) and the Comité National de Lutte contre la Pratique de l'Excision (CNLPE) within the Ministry of Social Action and National Solidarity, the government's body coordinating activities against FGM/C. The study has three components. The first describes the institutional, political and programmatic environment in Burkina Faso; the second is a secondary analysis of data from existing studies; and the third explores the current perceptions and behaviours of communities, and identifies the factors that contribute to the abandonment or preservation of the practice.

\section{Methodology}

The first component begins with a review of the history of the main events in the campaign against FGM/C, and then reviews the international conventions to which Burkina Faso subscribes, as well as the national texts adopted in the field of human rights, and specifically the rights of women and children. It also analyses how criminal prosecutions have been undertaken since the law against FGM/C was adopted. Finally it considers several intervention programmes that have been set up and notes the gains achieved and the limits to further successful action.

The review collected documents relating to international and national texts and laws, and assembled documents and reports of research studies and evaluations from several anti-FGM/C programmes. Interviews were conducted with staff from several ministerial institutions: the Ministries of Social Action and National Solidarity (MASSN), of Health, Justice and of Education; with NGOs and national associations, and with development partners. In total, 28 stakeholders were interviewed and three courts of justice were visited to observe cases in which judicial penalties were imposed. The interviews focused on the strategies employed, such as political persuasion and community mobilisation, evaluation of interventions that had been carried out, how the law has been applied, cooperation between government departments, and the prospects for a more effective campaign against $\mathrm{FGM} / \mathrm{C}$, as well as recommendations to the CNLPE to make their activities more effective.

The second component involved a secondary analysis of existing data and comparisons of the trends in FGM/C over the past ten years. The characteristics of women whose daughters had been excised were compared with those of women whose daughters had not been excised, and the principal factors which affect the risk of girls being excised or not were measured. Altogether, eleven quantitative studies and five qualitative studies were analysed. 
The third component was a qualitative survey of the current perceptions and behaviour of the communities. This involved an examination of whether the changes that are recorded in the studies reflected a real change in social behaviour or simply resulted from incorrect declarations made because of fear of punishment. The ethnographic approach of participant observation was used, which involved researchers immersing themselves in the daily lives of communities in five provinces, using free informal discussions and more structured discussions. Sampling was based on an equitable distribution by area; within each area considered, focus groups and individual discussions were conducted in several localities. Altogether, 85 in-depth interviews and 44 focus groups were arranged and recorded.

Each component has resulted in a separate report. This document provides a synthesis of the main results of the three studies.

\section{Results}

\section{A firm political will}

Burkina Faso has for many years enjoyed strong governmental support for ending FGM/C . In 1983, during the revolution period lead by President Sankara, concern over FGM/C was endorsed by the government. The creation of the National Committee for the Campaign against Excision (CNLPE) in 1990, and of its permanent secretariat in 1997, as well as the assumption of responsibility for its budget, marked a decisive turning point in the campaign against FGM/C. Adoption of the law banning the practice in 1996 and its subsequent application have been key factors in this process. The running costs of the CNLPE and of the 'green telephone' (a telephone helpline) have been funded from the national budget. Burkina Faso has ratified various conventions, which have provided a framework for interventions in the campaign against FGC.

\section{A multiplicity of interventions}

Anti-FGM/C activities in Burkina Faso are varied and concern several areas: community leaders as well as religious and political leaders, the security forces, the judiciary, medical personnel, teachers, youth associations, women's associations and journalists. This involvement of various categories of society has increased the willingness of socio-professional groups to engage in the campaign against FGM/C. The CNLPE has made a tremendous effort to involve diverse professional groups and influential persons in society in the campaign. The media have been used at every stage in the process. Successes include the institution of a green telephone line "SOS Excision," capacity-building among socio-professional groups, persuasive communication messages involving the wife of the President, lobbying, an IEC campaign, the provision of remedial medical help for those who have undergone excision, mobile security teams to increase awareness of and to reinforce the law, and the inclusion of FGM/C in existing development and reproductive health programmes involving adolescents or women.

Despite limitations because messages were not always adapted to specific target audiences, difficulties involved in imposing the penalties that are provided for by the law, and a lack of human and financial resources, Burkina Faso has succeeded in achieving and sustaining a remarkable level of activity against the practice. 


\section{Quantifiable changes}

Even though most of the existing studies on excision do not cover the entire nation, it is still possible to conclude that there is a lower prevalence rate among girls in the age group $0-10$ years compared to older girls and adult women. The difference between daughters and their mothers is higher than 50 percent and this difference is increasing over time. The main ethnic groups in which girls are at risk of being cut are Peul, Bobo and Samo. Daughters of mothers belonging to these ethnic groups have between 2.4 to nearly 7 times a greater risk of being excised compared to daughters whose mothers come from Mossi, Goin-Senufo, Lobi-Dagara and Gourmanche ethnic groups. Girls belonging to the Gourounsi ethnic group have an even lower risk of being excised.

Place of residence and occupation are also important factors. Girls who live with their mothers in rural areas have 1.2 times the risk of being excised than girls who live in urban areas. This is probably because information circulates more readily in towns and the level of education is higher there, which helps girls to escape being excised. Education invariably seems to be a factor that reduces the risk of a girl being excised, especially where the mother has had at least secondary education. As far as occupations are concerned, it appears that for a girl to have a mother who is a government employee or an office worker means a lower risk of being excised. Belonging to the Muslim religion increases the risk for a girl to be excised, compared to those who follow other religions.

The prevalence throughout the nation as a whole, according to DHS data, still remains high, despite all these developments. However the older generations (those between 20 and 49) show a higher level of prevalence than those of younger generations. Among women between 15 and 19 years of age, the level is over 50 percent, whereas for girls of less than 5 years of age, the level has varied around 20 percent. In some provinces, such as Bazega, the level is under 10 percent.

Nowadays, very few men and women openly support FGM/C. In some provinces, only three percent of people declare support for the practice, and fewer than six percent of men and women still want to have existing or future daughters excised.

\section{Confirmation of the abandonment of FGM/C and factors that affect the changes}

The qualitative survey carried out in five provinces confirms that the practice of FGM/C is gradually being abandoned in all the areas visited. The communities in all these areas have full knowledge of FGM/C, are able to draw distinctions between the different kinds of cutting, and are well aware of the connection between excision and its consequences for health, including long-term consequences.

The information collected shows how significant the impact of the different strategies and activities has been in persuading people to abandon the practice, leading to widespread declarations of abandonment. Abandonment of FGM/C is not something recent, and mostly coincides with the time of adoption and application of the law. This period also corresponds with the beginning of a series of reinforcing strategies against excision. Some communities reported that an increasing number of young girls are no longer being excised and some of these are already reaching marriageable age. This is confirmed by agents of the Ministry of Health 
working in various health facilities reporting that they are seeing an increasing number of young pregnant women or new mothers who are not cut.

In terms of attitudes, communities stated that a critical mass ready to declare abandonment of the practice has already been reached. In all regions, songs and dances celebrating the girl who has been excised seem to have disappeared. There is a widespread view among many people, however, that even if the number of girls who have not been excised is increasing, this does not yet translate into a broader demographic impact.

\section{Factors that have contributed to the abandonment of excision}

The anti-FGM/C law is well known to most people, especially the punishments for those found guilty. A strategy of denunciation carried out by CNLPE is equally well-known and continues to arouse fear among people, which has been a significant force in changing opinions. The restrictive and compulsory aspect of the law is extremely visible, and the setting up of mobile security teams for dissuasion and communicating information has also contributed to reducing the practice.

The involvement of certain traditional authorities in abandoning the practice of excision has received popular support. This has enabled a distinction to be drawn between justifications based on traditional custom for maintaining excision and other types of justification.

Continuous and diverse intervention, including the involvement of several sectors and of NGOs, as well as the media in the campaign, is a key element affecting people's awareness and willingness to change.

Contacts with the outside world and with alternative values have also contributed to a change in behaviour. They have helped new ideas to be absorbed and have confirmed the decision to give up an old practice. It is widely accepted that the level of education and social status are major factors in the campaign against excision. A change in norms and values has thus resulted from contact with the outside world, with modern ideas being introduced and with education. This has led to the creation of an environment where FGM/C no longer has the traditional value it used to have.

Living with and seeing the consequences of excision have been, for some people, a major step in their life. These consequences have helped them to accept the real dangers of excision for small girls and have provided a powerful stimulus for changing their outlook towards excision and for their determination not to have their daughters excised.

Economic disadvantages are also mentioned; when faced with health problems resulting from FGM/C, people see more clearly the economic problems created for couples when the mother comes to give birth.

Belonging to the Christian religion, particularly Protestantism, is a significant factor in abandonment of excision. 
Seeing the sequelae of FGM/C is another important factor. Several people reported having changed their mind when they saw, in their immediate family or neighbourhood, harmful consequences of the practice. These consequences range from haemorrhage to long-term disabilities. The programme to provide medical care for those suffering health consequences has also increased testimony from women on the harmful effects of FGM/C.

Implementation of the decision not to cut daughters is rarely made public; it is more a private and family decision. Other members of the family may not understand this position, which can result in social isolation, threats to denounce family members who want to continue the practice, and decisions to isolate their daughters from the village.

\section{Continuing resistance to change}

Even if change is real, common and visible, there is still some resistance among certain groups. The depth of their convictions encourages some supporters of excision to take risks in continuing to practice it, in spite of the law. In addition to these risks, the use of subterfuge and swearing to secrecy and utmost discretion, as well as a degree of complicity, are essential for such support to continue. Cohesion within a group is an important factor enabling those with firmly held convictions to perpetuate them. Certain areas (e.g. Gourma) or certain ethnic groups (e.g Ouoba) seem more resistant than others; even so, in all the areas where research has been conducted, open expressions of resistance have become uncommon.

Clandestine cases of excisions are reported. To achieve their aims, those resisting change have developed strategies to escape the attention of the authorities. Perpetuation of the practice by these groups is carried out by changing the usual timing for excision and the age at which it is carried out, so that cutting young girls less than five years old is preferred because they are more docile. The rainy season and night-time are often used so that activities can be more easily concealed.

Certain beliefs persist that continue to convince some people of the need to sustain the practice. For examples, the clitoris is considered to be an organ which can keep on growing and increases the degree of masculinity in a woman - and it absorbs the blood of the foetus during childbirth, which is said to explain why some women cannot bear children. It is still regarded as a source of illness that creates a permanent state of sickness in a woman. The consequences of non-excision for the sexuality and the behaviour of an uncut woman are very evident in what is said by those holding such resistant views.

\section{Conclusion}

The creation of a favourable environment for the campaign against excision in Burkina Faso has facilitated various anti-cutting activities over the past two decades. Many studies have been conducted that have enabled co-ordinated activities to be better designed and carried out. Various international agreements have been signed, giving an international character to actions and activities developed by the national government for promoting abandonment. The adoption of the law against excision and its effective implementation have been crucial factors for Burkina Faso. The firm political will of the government, which has spared no effort in supporting the campaign, has enabled a holistic vision that incorporates all socio-professional categories. The media have been constantly used throughout the whole process to disseminate and advocate the 
position of the government and other stakeholders. The continued actions of CNLPE and its partners have greatly helped in raising popular awareness of the dangers of excision and impacted the abandonment of the practice. All these actions have enabled the achievement of gains, both in terms of awareness and behaviour change.

We must, nevertheless, concede that some of the strategies need further readjustment, including:

- Provision of fresh training for new generations of police, lawyers, and judges to enable strict application of the law

- Intensifying submissions to development partners for increased financing

- Greater use of the media, particularly community radio stations

- Sharper focus for strategies, including new strategies that take into account psycho-sexual aspects of FGM/C and the stigmatisation of girls who have not been excised

Among the reasons why excision still continues in Burkina Faso is a certain view of the world and a model for women within the community, which includes FGM/C as a guarantee of sexual stability, of virginity and chastity, of fidelity within the home and of successful marriage. But even if social and family pressures remain strong, the mechanisms for maintaining this practice socially are now diluted within most communities, and so sustaining the practice has become largely an individual and family responsibility. This is clearly an important achievement and major change that the CNLPE and its partners should build on and exploit further to accelerate abandonment of the practice over the coming decade.

Following is the full version of the above-summarized report in the original French. 


\section{Table des matières}

Introduction ...................................................................................................................................................

Objectif........................................................................................................................................................8

Methodologie ......................................................................................................................................................9

Présentation des modèles théoriques..................................................................................................13

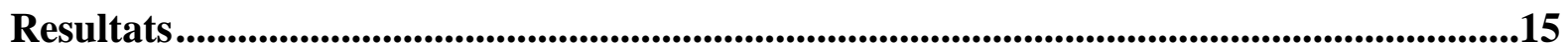

L'évolution institutionnelle et l'environnement juridique .........................................................................15

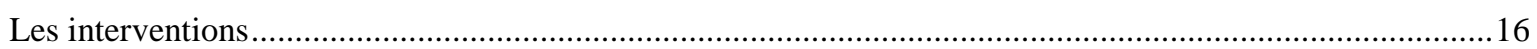

Le Comité National de Lutte contre la Pratique de l'Excision .....................................................................17

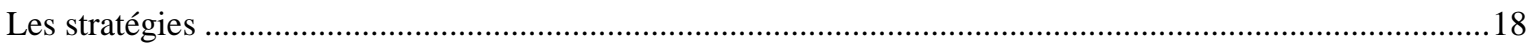

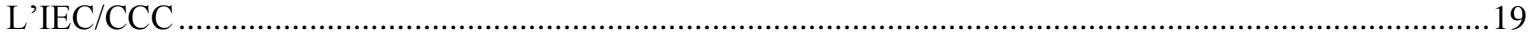

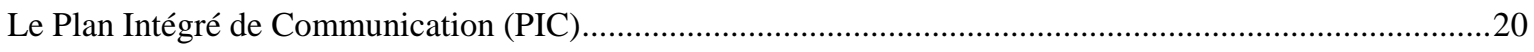

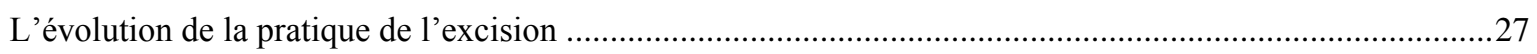

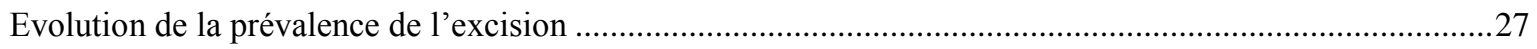

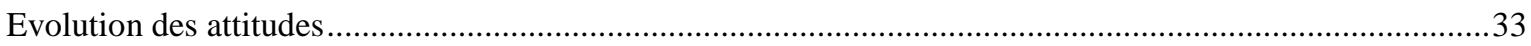

Analyse des perceptions et comportements des communautés .......................................................................34

Les contraintes à l'adoption de la non pratique de l'excision......................................................................40

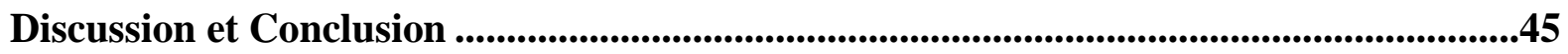

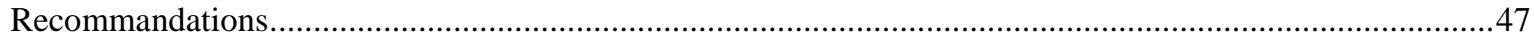

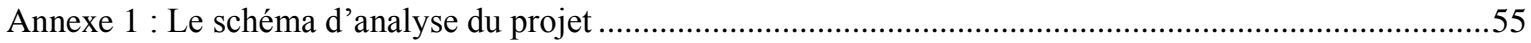




\section{INTRODUCTION}

L'excision touche un grand nombre de femmes et de filles dans le monde, mais plus particulièrement en Afrique où elle est pratiquée dans une vingtaine de pays. Au Burkina la pratique touche l'ensemble du pays et concerne toutes les religions et presque tous les groupes ethniques. La forme d'excision la plus pratiquée est celle qui consiste en l'ablation du clitoris et des petites lèvres.

Depuis quelques décennies, il existe une réelle prise de conscience face à la pratique de l'excision, dans le cadre de l'amélioration des conditions de vie des femmes et des enfants, notamment de la petite fille. Celle-ci s'est traduite par un engagement politique fort avec l'adoption de plusieurs actes politiques et administratifs. En plus de la mise en place d'un comité national et la poursuite de ses activités, de la mise en application des mesures coercitives, plusieurs programmes d'intervention sont mis en œuvre pour mettre fin à la pratique.

Plusieurs recherches sur la santé de la reproduction et sur l'excision permettent de penser que cet environnement politique et administratif et les programmes d'intervention ont permis d'influencer l'approbation pour la pratique et les comportements.

Cependant les résultats de la prévalence nationale de l'EDS ne font pas apparaître de changement. Aussi il est nécessaire de se demander si la baisse déclarée dans certaines études est le reflet d'un changement social réel ou si elle est le reflet de fausses déclarations dû à la peur de réprimandes. Pour certains, la pratique serait rentrée dans la clandestinité, tandis que pour d'autres elle reflèterait un réel déclin.

Si la baisse de la pratique est réelle, il y a lieu de s'interroger sur les facteurs qui y ont contribués: les types d'intervention qui ont plus d'effet, le rôle de la volonté politique et l'application de la loi. Il se peut également que ce soit l'effet de facteurs endogènes tels que l'éducation ou l'influence de l'instruction et de la culture moderne.

\section{OBJECTIF}

Cette étude avait pour objectif général de faire une analyse de la situation de l'excision au Burkina afin de tirer des conclusions pour le processus politique et programmatique de lutte contre la pratique en Afrique. Spécifiquement il s'agissait de :

- Evaluer les tendances de la pratique de l'excision au courant des 10 dernières années ;

- Identifier les perceptions et les facteurs qui justifient l'arrêt de la pratique ou sa continuation ;

- Identifier les facteurs politiques, programmatiques et endogènes qui peuvent expliquer la discontinuité de la pratique ;

- Mesurer le degré de réceptivité des messages de lutte contre la pratique de l'excision par les communautés ;

- Identifier les stratégies utilisées par les populations pratiquant l'excision. 


\section{Methodologie}

Dans le but de faire une analyse rigoureuse et compréhensible de la situation, l'étude a suivi le schéma analytique décrit dans un diagramme en annexe. Au total, quatre méthodes ont été utilisées pour collecter et analyser les données : l'analyse secondaire des données des études déjà réalisées sur l'excision au Burkina, la revue documentaire des actions politique, légale et administrative en matière d'interventions contre l'excision, la revue et l'analyse de quelques programmes d'intervention et enfin, l'enquête sur les perceptions et facteurs influençant ou non l'abandon de l'excision.

1. L'analyse secondaire des données d'étude. Elle a pour objectif de voir l'évolution des indicateurs au niveau provincial, régional et national et de dégager les niveaux et les tendances. Toutes les données collectées depuis 1994 sur le plan national ont été rassemblées, réparties en deux catégories (quantitative et qualitative).

Tableau 1 : Etudes réalisées depuis 1996

\begin{tabular}{|l|l|l|l|}
\hline De 1996 à 2000 & Couverture & Après 2000 & Couverture \\
\hline \multicolumn{3}{|c|}{ Etudes quantitatives } \\
\hline INSD (1996) & Nationale & Sangli Gabriel et Ouédraogo Moctar (2001) & 7 provinces \\
LSC 1996 & 1 province & Ouédraogo ldrissa et al. (2001) & Nationale \\
LSC 1998 & 1 province & Kaboré Yimian et al. (2002) & 16 provinces \\
EDS 98/99 & Nationale & EDS 2003 & Nationale \\
Ouédraogo Diénéba et al. (2000) & 5 provinces & PEBC 2001 & 1 province \\
& & PEBC 2003 & 1 province \\
\hline \multicolumn{3}{|c|}{ Etudes qualitatives } \\
\hline Konaté Alimata, 1993 \\
Tougry Ester, 1998 & \multirow{2}{|c|}{ Yé minata, 2000 } & 2 départements \\
\hline
\end{tabular}

Sur l'ensemble des 11 études quantitatives, quatre sont d'envergure nationale et quatre autres ont eu lieu dans une seule province. Trois études ont connu au moins un second passage. Les études qualitatives sont circonscrites au niveau village ou département et ne sont pas répétées.

L'analyse des données quantitatives met l'accent sur les changements intergénérationnels dans la pratique (avec une attention particulière pour les filles de moins de 10 ans) et les attitudes (approbation, approbation du conjoint, regrets de l'excision des filles, intentions futures, approbation de la loi). Pour déterminer les facteurs influençant la prise de décision au niveau des individus, une analyse multivariée a été menée avec des variables indépendantes telles que l'instruction, la résidence, le type de famille et le niveau économique; l'hypothèse étant que le profil de ceux qui ne pratiquent pas l'excision informera sur la dynamique sociale et les changements contemporains et facilitera les prédictions sur les tendances futures.

L'analyse qualitative des données secondaires se focalise sur les facteurs socioculturels, économiques et psychologiques de la pratique de l'excision. Elle a permis aussi d'identifier les questions sur lesquelles élaborer de nouveaux questionnaires pour l'enquête sur les facteurs 
influençant l'abandon de la pratique. (Rapport Analyse Secondaire des Données d'Enquête 19962004, Ouagadougou, septembre 2005, 22 p.).

2. La revue documentaire de la politique nationale, légale et administrative en matière d'interventions contre l'excision. Cette revue documentaire essaye de déterminer l'étendue du rôle du politique en termes de facilitation ou de stimulation de changement. Deux approches ont été utilisées : la revue des documents politiques et l'évaluation de l'application des mesures juridiques.

La revue des documents politiques s'est intéressée aux différentes conventions et chartes internationales et sous régionales relatives à la pratique de l'excision qui ont été adoptées par le Burkina. Elle a permis une revue de la politique courante, législative, juridique et administrative à propos de l'excision en analysant les documents gouvernementaux, ceux des donateurs et organismes internationaux et permet d'apprécier les étapes de mise en œuvre des actions contre l'excision.

L'évaluation de l'application des mesures politiques est suivie par la revue des mises en œuvre par le système administratif. Les textes décrivant l'application des mesures sont examinés et les institutions responsables de la mise en application ont été visitées au niveau national et provincial où des discussions approfondies avec des techniciens de terrain ont eu lieu dans le but d'évaluer leur perception de l'effectivité de la mise en œuvre des politiques. Au niveau national, des représentants des institutions ci-dessous ont été interrogées.

Tableau 2: Institutions visitées et nombre de personnes ressources interviewées

\begin{tabular}{|l|l|l|l|l|l|l|l|}
\hline Institutions & $\begin{array}{l}\text { Ministère de } \\
\text { l'Action } \\
\text { sociale }\end{array}$ & $\begin{array}{l}\text { Ministère } \\
\text { Santé }\end{array}$ & $\begin{array}{l}\text { Force } \\
\text { sécurité }\end{array}$ & $\begin{array}{l}\text { Ministère } \\
\text { Justice }\end{array}$ & $\begin{array}{l}\text { Ministère } \\
\text { Education }\end{array}$ & Partenaires & ONGs \\
\hline $\begin{array}{l}\text { Personnes } \\
\text { ressources }\end{array}$ & 4 & 3 & 9 & 3 & 2 & 3 & 4 \\
\hline
\end{tabular}

Rapport Analyse de l'Environnement Institutionnel, Politique et Programmatique de la Lutte contre l'Excision au Burkina Faso, Ouagadougou, mars 2006, 30 p.

3. L'analyse de quelques programmes d'intervention. Cette revue a permis de s'entretenir avec quelques acteurs clés pour examiner les interventions et recueillir de la documentation sur les changements obtenus au niveau communautaire. Ce travail a permis d'identifier les succès et les limites des stratégies.

Vingt huit responsables de programmes ont été interviewés. Dans la plupart des cas, les interviews individuelles ont été enregistrées, avec l'accord du répondant. Pour chaque type de répondant, des instruments ont été élaborés. Des enquêteurs ont été recrutés pour collecter les données auprès de certains responsables et personnes ressources de structures au niveau national et auprès des partenaires au développement. (Rapport Analyse de l'environnement institutionnel, Politique et Programmatique de la lutte contre l'excision au Burkina Faso, Ouagadougou, mars 2006, 30 p.) 
4. L'enquête sur les perceptions et les facteurs influençant ou non l'abandon de la pratique.

C'est une collecte des données auprès de plusieurs communautés. Elle tient compte des zones où des interventions ont été mises en œuvre dans le but d'accélérer le processus de changement social. Deux modèles ont été utilisés afin de préparer la collecte et l'analyse des données de terrain : la carte mentale ${ }^{1}$ et le modèle de diffusion de l'innovation ${ }^{2}$.

Cinq zones ont été choisies pour cette collecte de données (Tableau 3). Plusieurs critères ont prévalu au choix de ces zones et localités : la langue parlée, la situation géographique, le niveau de prévalence de l'excision, la mise en œuvre de programme d'intervention, le type de programme d'intervention et la proximité.

Tableau 3 : Localités d'enquête

\begin{tabular}{|l|l|l|l|}
\hline \multicolumn{2}{|c|}{ Zones (ou provinces) } & \multicolumn{2}{l|}{ Localités (villages et/ou quartiers) } \\
\hline Oudalan & Korézena & Saouga & Débéré Naguel \\
\hline Houet & Accart-Ville & Yéguéré & Colma \\
\hline Gourma & Koaré & Momba & Nayouri \\
\hline Kadiogo & Bilbalogo & Tanghin (sect. 23) & Secteur 30 \\
\hline Zoundwéogo/ Bazèga & Béré & Bindé & Toécé \\
\hline
\end{tabular}

Cette étude essentiellement qualitative a privilégié l'immersion ou l'observation participante à cause de la nature délicate de la question. Pour ce faire, une mission de prospection a sillonné les différentes localités pour nouer des contacts, répertorier les personnes ressources et préparer la venue des agents de collecte des données. Une immersion de un mois a été retenue pour les agents de collecte de l'information. Cette phase d'immersion a utilisée les techniques de l'observation participante. Les enquêteurs faisaient un rapport journalier tous les soirs sur les informations pertinentes recueillies. Cette immersion a aussi permis d'identifier toutes les personnes susceptibles de fournir des informations et surtout d'identifier les personnes favorables et non favorables à la pratique de l'excision.

Suite à cette phase, des guides d'entretien plus structurés ont été élaborés pour les entretiens semi-ouverts informels et les entretiens structurés. Plusieurs méthodes de collecte de données ont été utilisées : le récit de vie, l'entretien individuel approfondi et l'entretien de groupe focalisé (focus groups). Ces entretiens ont tous été enregistrés, transcrits, saisis et entrés dans le logiciel NUD*IST ${ }^{3}$ pour l'analyse.

Parmi les personnes ressources, il y avait les agents de santé et les enseignants d'écoles. Bien qu'étant en général des "non natifs" dans les localités, le fait d'y résider depuis plusieurs années et d'appartenir à des services sociaux en font des informateurs clés. Avec les agents de santé, il s'agissait de collecter des informations sur la situation sanitaire, l'observation du statut des fillettes et des femmes en consultation prénatal ou pendant l'accouchement.

\footnotetext{
${ }^{1}$ World Health Organization. 1999. What works and what doesn't work

${ }^{2}$ Rogers Everett, 1995 : Diffusion of Innovations. Fourth edition, New York, NY : The Free Press
} 


\section{Les entretiens structurés}

Plusieurs méthodes de collecte de données ont été utilisées : le récit de vie, l'entretien individuel approfondi et l'entretien de groupe focalisé (focus groups). Ces entretiens ont tous été enregistrés.

- Le récit de vie : ils ont été réalisés avec les ex exciseuses, des filles/ femmes excisées portant ou non des séquelles, des filles femmes non excisées. Les récits de vie des filles/ femmes visaient à recueillir leurs différentes expériences en matière de vécu de l'excision ou de la non excision, de déceler en quoi cette expérience peut être porteuse de changement. Il s'agissait aussi de connaître l'expérience des exciseuses, leurs expériences d'abandon ou de poursuite de la pratique dans le contexte de la loi.

- Les focus groups : trois guides ont été élaborés. Il s'agit du guide pour les élèves ; guide pour les jeunes (filles et garçons) âgés de 15-24 ans; guide pour les adultes (femmes et hommes) de plus de 25 ans.

- Les entretiens individuels : plusieurs supports de collecte des données ont été élaborés. Il s'agit notamment du guide d'entretien individuel avec les leaders d'opinion favorables à la pratique de l'excision; guide d'entretien individuel avec les leaders d'opinion non favorables à la pratique de l'excision; guide d'entretien individuel avec les personnes ressources favorables à la pratique de l'excision; guide d'entretien individuel avec les personnes ressources non favorables à l'excision.

Ces différents groupes ont permis de trianguler les informations collectées aussi bien en entretiens informels qu'en entretiens structurés.

Pendant toute la période de collecte des données, les agents de collecte avaient l'obligation de consigner toutes les informations recueillies pendant l'observation et les entretiens non structurés dans un cahier appelé «cahier d'immersion ». Pour ce qui concerne les entretiens individuels structurés et les focus groups, ils ont été pour la plupart enregistrés et retranscrits. Le consentement écrit ou verbal des informateurs était une étape obligatoire avant le début de tout entretien structuré.

Le fait de résider dans la localité durant toute la période de la collecte a permis de tisser des relations de confiance qui ont dissipé les craintes manifestées au début. Plusieurs rencontres étaient parfois nécessaires avant la proposition d'enregistrement. Aussi les entretiens que l'enquêteur sentait être trop conformiste, était repris quelques jours plus tard lorsque la confiance était rétablie. Pour établir la confiance, l'enquêteur participait parfois aux activités de l'enquêté (maraîchage, cuisson des repas, pilage de mil, puisage d'eau au puits, ramassage de bois, etc.) ou s'attachait les services d'une tierce personne en qui l'enquêté avait pleinement confiance pour l'assurer de la confidentialité des informations à collecter. Les entretiens trop conformistes étaient également conservés pour les éventuels besoins de comparaison entre le premier et le second discours. Lorsque malgré tout l'enquêteur constatait que l'enregistreur était la principale difficulté à surmonter, l'entretien complémentaire se passait sans enregistreur. Les informations étaient ainsi reportées dans les cahiers d'immersion. 
La collecte a duré un mois et a permis de réaliser au total 44 focus groups enregistrés et 85 entretiens individuels enregistrés auprès de plusieurs personnes : personnes favorables et non favorables à l'excision, filles excisées et non excisées, ex-exciseuses, leaders d'opinion, responsables coutumiers et religieux, personnes ressources telles que les agents de santé, enseignants, responsables et membres d'associations, d'écoles, personnel de soutien des centres de santé. Toutes les personnes interrogées ont été identifiées pendant la phase d'immersion, période pendant laquelle les 15 agents de collecte, répartis en 5 équipes, ont séjourné durant tout le mois dans leur localité respective. Les résultats complets sont présentés dans un rapport. (Analyse de la Réponse Communautaire, Ouagadougou, septembre 2006, 62 p.).

\section{PRESENTATION DES MODELES THEORIQUES}

Afin de mieux appréhender le processus de changement social, deux cadres conceptuels ont été utilisés : la carte mentale et le modèle de diffusion de l'innovation. Le premier met l'accent sur le rôle et la représentation de l'excision dans la société en identifiant les raisons et les mécanismes de renforcement communautaire. Le second quant à lui, met l'accent sur les facteurs et le processus de modification du comportement face à une quelconque innovation, aussi bien dans la continuité des comportements anciens que dans l'adoption de nouveaux types.

\section{La carte mentale}

Cette carte met en exergue les raisons psychologiques et sociales ainsi que les convictions religieuses, sociétales et individuelles (hygiène et esthétique) contribuant à la pratique de l'excision.

Ces convictions recouvrent notamment : la poursuite des coutumes et des traditions, la protection de la propreté, de la chasteté et de la virginité, la sauvegarde de l'honneur familial, la nécessité religieuse et le contrôle de la sexualité des femmes pour la protection de la communauté dans son ensemble.

\section{Graphique 1 : La carte mentale}

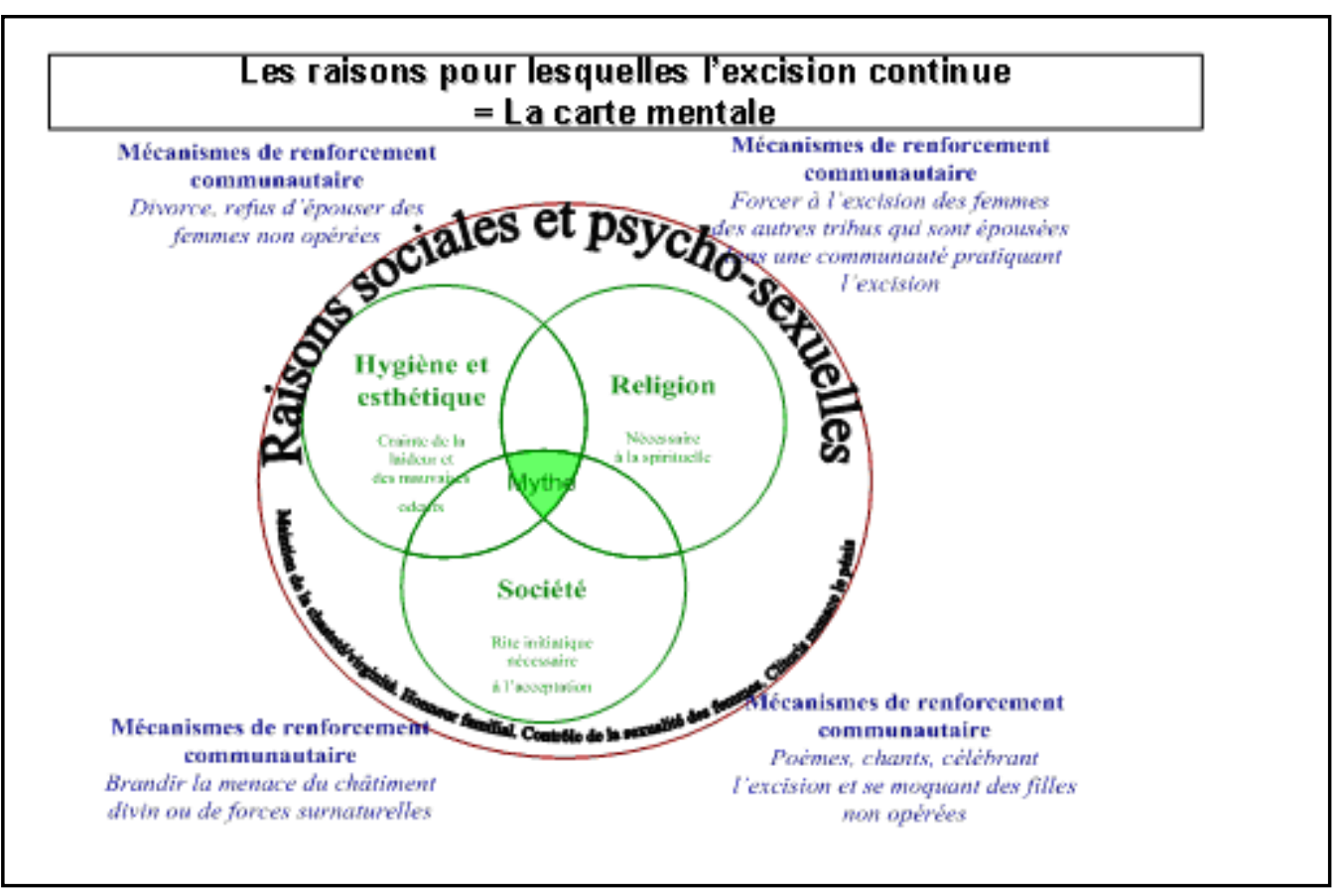




\section{Le modèle de Diffusion de l'Innovation}

Le modèle de «diffusion des innovations » a été utilisé pour guider la collecte des informations sur un éventuel changement de comportement au niveau communautaire. C'est un processus par lequel un individu (ou une autre unité de prise de décision) passe d'une première connaissance d'une innovation à la formation d'une attitude à l'égard de l'innovation. Par la suite, il passe à l'étape de décision d'adoption ou de rejet, à la mise en application de la nouvelle idée et à la confirmation de cette décision. La diffusion est définie comme le processus par lequel (1) une innovation (2) est communiquée à travers des chaînes de réseaux (3) au-delà du temps (4) au sein des membres d'un système social.

$\mathrm{Ce}$ modèle suggère que les nouveaux concepts et comportements ne soient pas adoptés par tout le monde à un seul moment donné dans le temps, mais ils sont plutôt adoptés d'abord par des «innovateurs » ou précurseurs. $\mathrm{La}$ diffusion se produit graduellement dans une communauté, jusqu'à ce qu'une «masse critique » de partisans soit atteinte; et c'est alors que la diffusion pourrait s'accélérer pour gagner le reste de la communauté.

Il s'agissait d'identifier en premier lieu les «innovateurs» ou « précurseurs » et explorer le fait que certaines communautés devraient avoir évolué beaucoup plus rapidement que les autres. Dans ces conditions, il serait important de chercher à comprendre les niveaux et les étapes des communautés dans le processus de changement. Plusieurs questions sous tendent ce travail: estce le début du processus où les précurseurs ont besoin de soutien pour influencer les normes de comportement ou est-ce un stade plus avancé ? Quelles conditions déterminent l'adoption et comment le consensus est-il obtenu ? Quel est le temps nécessaire pour atteindre le point critique de l'abandon? Existe-t-il une hiérarchie dans le processus d'adoption des nouvelles idées et comportement? 


\section{RESULTATS}

\section{L'évolution institutionnelle et l'environnement juridique}

L'évolution institutionnelle et juridique actuelle de la lutte contre l'excision au Burkina résulte de la combinaison des actes administratifs nationaux et du cadre juridique national et international dans lequel s'inscrit le pays.

\section{Historique}

L'environnement favorable à la lutte contre l'excision au Burkina est marqué également par un certain nombre d'actions qui peuvent être divisées en quatre périodes : les années 1900 à 1984, la période révolutionnaire à la veille de la $4^{\text {ème }}$ république, la décennie 1990 et la période après 2000.

- Pendant la colonisation, les missionnaires catholiques ont tenté de mettre fin à l'excision en menaçant d'excommunier les contrevenants, sans pouvoir apporter de réponse à la question du mariage des filles nouvellement non excisées. En 1960, la résistance farouche des dignitaires du régime et de la chefferie traditionnelle et coutumière a eu raison de la tentative de la première république de mettre fin à la pratique. En 1975, on assiste à la première annonce radiophonique, d'informations tendant à discréditer la pratique de l'excision, lors de la célébration de la journée internationale des femmes.

- Quatre mois après l'avènement de la révolution (le 4 Août 1983), Edmond Kaiser de l'ONG « Terre des hommes » rencontre Thomas Sankara, président du Burkina Faso, sur la question de l'excision. En 1985, un séminaire est organisé par l'Union des Femmes Burkinabé (UFB), pour parler des questions liées à la femme, y compris l'excision. Au cours de ce séminaire, les femmes ont demandé l'adoption de textes interdisant purement et simplement la pratique de l'excision. En 1988, sous le Front Populaire, un séminaire d'envergure nationale est organisé sur «les pratiques traditionnelles néfastes à la santé des femmes : le cas de l'excision ». Ce séminaire connaît la participation de plus de 300 délégués venus de toutes les provinces et de toutes les couches ou catégories de la population et est relayé par les différents média : radio, télé, presse écrite. Le comité d'organisation est érigé en comité national provisoire de lutte contre la pratique de l'excision.

- Le 18 mai 1990, le Comité National de Lutte contre la Pratique de l'Excision (CNLPE) est né sous un décret présidentiel (Kiti ${ }^{\circ}$ AN VII-318/FP/SAN-AS/SEAS) avec des démembrements dans les provinces et est composé de 60 membres nommés par décret présidentiel dont 13 ministères, plusieurs ONG et associations (féminines, professionnelles, des droits de l'homme), des leaders religieux, coutumiers ainsi que des personnes ressources. La présidence d'honneur est assurée par l'épouse du chef de l'Etat. En 1992, un premier plan d'action est adopté pour la période 1993 à 1997. Sur la base de ce document, une table ronde des bailleurs de fonds est organisée en 1994 et permet d'attirer l'attention et susciter l'engagement des partenaires au développement. A la faveur de la relecture du code pénal en 1996, la loi réprimant la pratique de l'excision est adoptée. En 1997, le comité national de lutte contre la pratique de l'excision se dote d'un 
secrétariat permanent, chargé de la mise en œuvre de ses activités et le gouvernement y affecte des agents de l'Etat (y compris l'engagement des forces de sécurité). Dans la même année, on assiste aux premières condamnations, principalement avec sursis, afin de sensibiliser la population dans un premier temps. Un second plan d'action est adopté pour la période 1999-2002.

- Depuis 2000, 1'excision est considérée comme faisant partie du programme d'investissement public et le budget de fonctionnement du secrétariat permanent est pris en compte dans le budget de l'Etat. Dans le même temps, la date du 18 mai est consacrée comme journée nationale de lutte contre l'excision dont la première a été célébrée en 2001. L'excision est aussi considérée comme un problème de santé publique par le ministère de la santé et un accent particulier lui est accordé dans la formation du personnel de santé.

\section{Les conventions et les textes sur les droits de la femme et de l'enfant}

Le Burkina Faso a ratifié plusieurs conventions internationales et adopté des textes au niveau national en faveur des droits de la femme et des enfants (Tableau 4).

\section{Tableau 4 : Conventions et textes adoptés}

\begin{tabular}{|l|c|}
\hline Les conventions et textes nationaux & $\begin{array}{c}\text { Date ratification } \\
\text { /adoption }\end{array}$ \\
\hline Conventions internationales & 1984 \\
\hline La charte de Banjul & 1987 \\
\hline La convention sur les droits des femmes & 1990 \\
\hline La convention sur les droits des enfants & 1992 \\
\hline La charte africaine & 1999 \\
\hline La convention sur les droits civils et politiques & 1999 \\
\hline La convention sur les droits culturels, économiques et sociaux & 1979 \\
\hline La charte africaine sur les droits de l'enfant & 2005 \\
\hline $\begin{array}{l}\text { Le protocole à la charte africaine des droits de l'homme et des peuples } \\
\text { relatifs aux droits des femmes (protocole de Maputo) }\end{array}$ & \\
\hline Textes nationaux & 1991 \\
\hline La constitution du Burkina & 1996 \\
\hline Le code pénal & \multicolumn{2}{|}{} \\
\hline
\end{tabular}

Ces conventions et textes fournissent un cadre de référence pour les interventions, mais il faut reconnaître qu'ils restent largement méconnus de la population.

\section{Les interventions}

Dans le but de contribuer à la réduction de la pratique de l'excision, plusieurs approches sont utilisées au Burkina. Deux plans d'actions nationaux ont été élaborés et mis en œuvre depuis 1996. Le Comité National de Lutte contre la Pratique de 1 'excision (CNLPE) en est l'organe de coordination. La mise en œuvre des interventions se déroule soit par le CNLPE, soit par des ONGs et associations. 


\section{Le Comité National de Lutte contre la Pratique de l'Excision}

Le Comité national de lutte contre la pratique de l'excision (CNLPE) est chargé de :

- La réalisation d'études et recherches en vue d'acquérir une connaissance adéquate du phénomène ;

- La collecte et la diffusion de toutes les données disponibles relatives aux pratiques affectant la santé des femmes et des enfants ;

- L'élaboration, la mise en œuvre et le suivi-évaluation des stratégies de lutte visant l'abandon de la pratique de l'excision et de toutes les autres formes de pratiques affectant la santé et l'épanouissement des femmes et des enfants ;

- La promotion d'activités de sensibilisation, d'éducation et d'information sur toutes ces pratiques ;

- La mobilisation des ressources matérielles et financières à la lutte contre toutes les pratiques affectant la santé et l'épanouissement des femmes et des enfants.

Au début de l'année 1997, le CNLPE s'est doté d'un Secrétariat Permanent (SP/CNLPE) pour rendre la structure plus fonctionnelle et plus opérationnelle. Ce secrétariat permanent est chargé de (i) la préparation des réunions et des documents du CNLPE ; (ii) la mise en œuvre et le suivi des décisions du CNLPE ; (iii) l'élaboration et la mise en œuvre du plan d'action national ; (iv) la gestion administrative et financière du CNLPE ; (v) la coordination des activités des structures décentralisées (CPLPE et CDLPE). Le gouvernement y affecte des agents de l'Etat, y compris l'engagement des forces de sécurité, afin d'assurer la disposition d'un personnel permanent.

Siège du Secrétariat Permanent

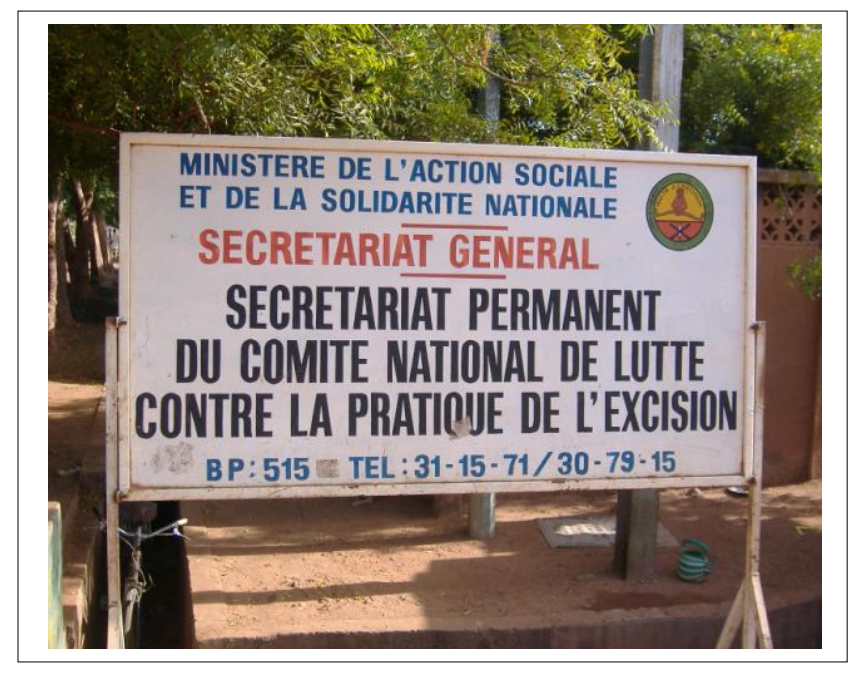

Le CNLPE est sous la présidence d'honneur de la première dame du Burkina et sous la responsabilité de la Direction du Ministère de l'Action Sociale et de la Solidarité Nationale; puis viennent le Secrétariat Permanent, ensuite les structures décentralisées composées de 13 cellules de Coordination Régionale et de 45 Comités provinciaux. Enfin, il existe un comité de soutien et des personnes ressources. 


\section{Graphique 3 : Organigramme du CNLPE}

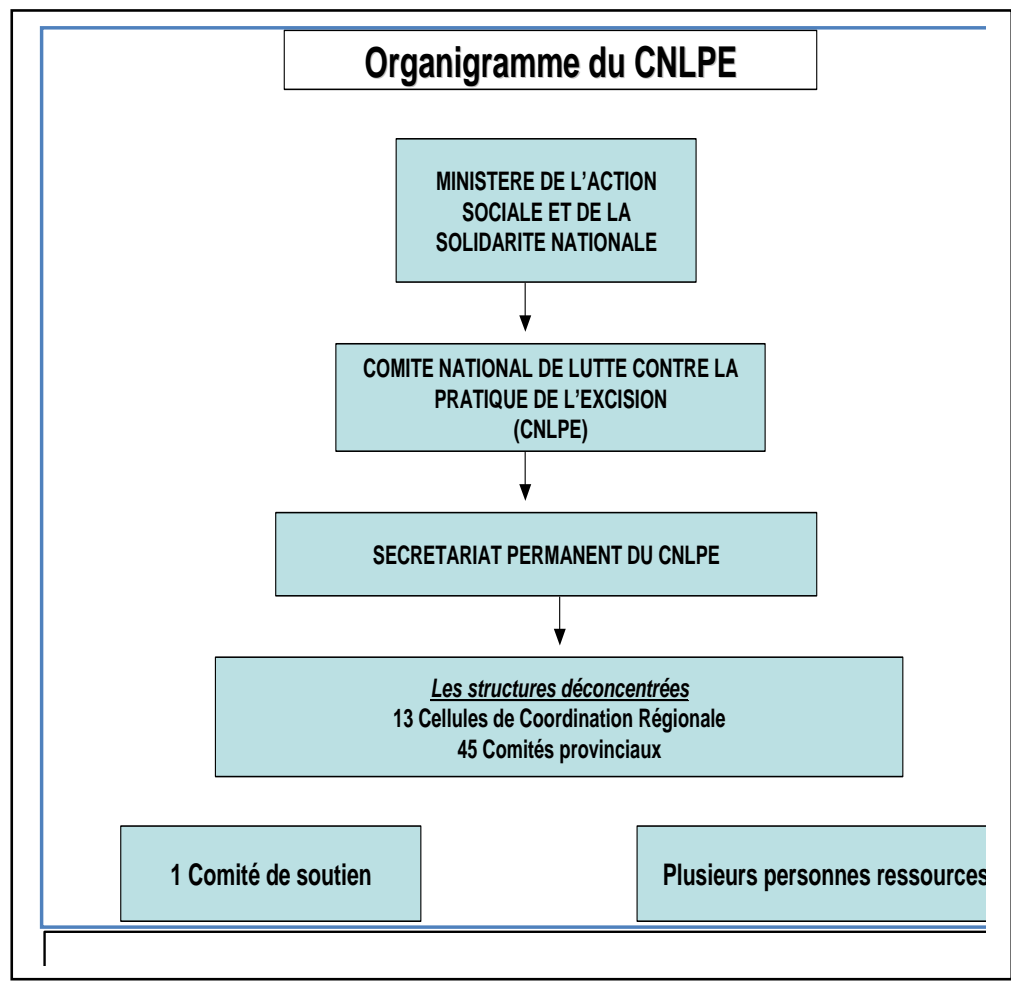

Treize ministères sont membres du comité, dont celui des droits humains, de la promotion de la femme et de la justice. Une collaboration étroite est assurée avec le ministère de la défense et la santé : la capacité d'intervention opérationnelle est traduite par la présence d'un personnel permanent de la gendarmerie au sein du secrétariat permanent du comité (pour accompagner et coordonner les interventions sur le terrain) et la réalisation des patrouilles de sensibilisation effectuées par 22 brigades de gendarmerie couvrant la majeure partie des zones identifiées comme à risque élevé. Avec le ministère de la santé, un agent de santé y est détaché pour coordonner les interventions médicochirurgicales en plus de deux gynécologues qui assurent la formation des autres gynécologues et autres médecins aux techniques de réparation mais accueillent également les patientes souffrant de séquelles. Tous les agents de santé ayant bénéficié de la formation sont chargés d'effectuer gratuitement les opérations de réparation dans les structures publiques de santé. L'acquisition d'un bloc opératoire dans la capitale permet d'y effectuer gratuitement les réparations. La collaboration avec le ministère de l'information contribue à la diffusion des informations et à la médiatisation des cas et des arrestations.

Depuis 1996, la loi est rentrée immédiatement en application, ce qui s'est traduit par les premières condamnations en 1997. Cette loi donne un cadre légal d'expression pour les défenseurs de l'intégrité physique de la petite fille et de la femme.

\section{Les stratégies}

\section{Le renforcement des capacités des acteurs.}

La formation est partie intégrante des actions. Plusieurs formations ont été réalisées. On notera celle des agents de santé, des magistrats, des avocats, des forces de police et de gendarmerie, des élèves, des leaders religieux, des leaders coutumiers, des députés, des enseignants du secondaire et du primaire, des exciseuses, des relais communautaires, des communicateurs traditionnels, et des journalistes.

Ces formations ont permis une meilleure capacitation et une plus grande prise de conscience des différents groupes socioprofessionnels. On assiste à une plus grande implication de toutes les couches de la population dans la lutte contre l'excision. 
Cependant, le manque d'intégration des modules sur l'excision dans la formation de base des écoles professionnelles (des enseignants, magistrats, avocats, médecins) ne permet pas un renouvellement systématique. De plus, le manque d'évaluation des activités après les formations ne permet pas de mesurer leur impact réel.

\section{L'équipement}

L'adhésion effective des partenaires au développement permet l'équipement du secrétariat permanent et des acteurs provinciaux: véhicules, motos, vélos, matériel audio - visuel, groupes électrogènes, supports d'animation, matériels.

\section{Le plaidoyer}

Les activités du plaidoyer sont menées envers les leaders religieux et coutumiers, les parlementaires, les responsables de groupements et associations à travers tout le pays. Il s'agit de rallier tous les leaders susceptibles d'influencer les attitudes et les comportements des populations, notamment les populations analphabètes. Au delà des objectifs du plaidoyer, la sensibilisation est effectuée continuellement pour une implication effective des leaders politiques, administratifs, religieux, coutumiers et traditionalistes avec création de comités de soutien des chefs coutumiers, des chefs religieux (catholiques et musulmans).

Les activités du plaidoyer ont permis l'institutionnalisation de la journée du 18 mai de chaque année comme journée nationale de lutte contre la pratique de l'excision par décret $\mathrm{n}^{\circ} 2001$ 258/PRES/PM/MASSN du 06 juin 2001, l'engagement de la société civile à travers l'implication des leaders d'opinion (coutumiers, religieux, traditionalistes), les Associations et les ONGs qui œuvrent à l'élimination de l'excision et sa prise en compte comme un problème de santé publique. Le plan d'action de lutte contre la pratique de l'excision est inscrit dans le programme d'investissement public du Ministère de l'Action Sociale et de la Solidarité Nationale.

\section{L'IEC/CCC}

L'approche principale mise en œuvre par le comité est l'information éducation communication (IEC). Plus tard, ce concept a fait place à la communication pour le changement de comportement (CCC). Cette stratégie utilise essentiellement les outils diversifiés comme support de communication pour impulser une conscientisation de masse, un changement d'attitude et de comportement.

On notera l'amélioration du niveau de connaissance des populations, une meilleure visibilité des activités d'intervention contre l'excision, une démystification de l'excision qui était considérée comme un sujet tabou.

\section{Les articles du code pénal condamnant l'excision}

Les trois articles suivants sont inscrits au chapitre II des crimes et délits contre la famille et les bonnes mœurs:

- Article 380

Est puni d'un emprisonnement de 6 mois à 3 ans et d'une amende de 150.000 à 900.000 francs ou de l'une de ces deux peines seulement, quiconque porte ou tente de porter atteinte à l'intégrité de l'organe génital de la femme par ablation totale, par excision, par infibulation, par insensibilisation ou par tout autre moyen.

$\mathrm{Si}$ la mort en est résulté, la peine est un emprisonnement de 5 à 10 ans.

- Article 381

Les peines sont portées au maximum si le coupable est du corps médical ou paramédical. La juridiction saisie peut en outre prononcer contre lui l'interdiction d'exercer sa profession pour une durée qui ne peut excéder 5 ans. 
Cette démystification s'est produite à travers les séances de sensibilisation et média qui utilisent les mannequins de femmes nues et les photos et images choquantes. La sensibilisation des magistrats a permis une meilleure application de la loi tandis que celle des forces de police et de gendarmerie a instauré la crainte d'être arrêté.

Cependant les messages sont trop généraux et ne s'adressent pas suffisamment à une cible spécifique. De plus, le caractère unidirectionnel des actions ne tient pas toujours compte de la remontée de l'information. L'absence de traduction du matériel de sensibilisation dans certaines langues limite l'appropriation des messages. Des zones reculées ont toujours un faible accès à l'information, par manque de couverture médiatique ou de financement conséquent.

\section{Le Plan Intégré de Communication (PIC)}

Le PIC est une approche de communication axée sur le plaidoyer, la mobilisation sociale autour de la question des mutilations génitales féminines et la communication pour le changement de comportement. La stratégie utilise les radios communautaires. Deux partenaires principaux contribuent à mettre en œuvre cette stratégie : l'UNICEF (dans 22 provinces) et l'Ambassade Royale des Pays Bas (dans 12 provinces).

Il y a une forte implication des autorités administratives locales, une adhésion des leaders d'opinion et une responsabilisation des acteurs locaux à travers les relais communautaires. On assiste à une forte implication des radios communautaires qui permettent une large couverture médiatique des messages sur l'excision, notamment en milieu rural avec une meilleure écoute des messages, parce que diffusés en langues locales. De plus, la sensibilisation au quotidien des relais communautaires assure l'information à tout temps. Les enregistrements portant sur des témoignages de cas de séquelles et de cas d'intention d'exciser sont constants.

Cependant la couverture du territoire national reste néanmoins limitée tandis que la période de diffusion des émissions n'est pas toujours adaptée au contexte local. Au niveau des relais communautaires, il y a parfois un faible contrôle dans leur choix, qui se reflète par un manque d'influence réelle dans la communauté. Les femmes y sont faiblement représentées. Les sensibilisations porte à porte ne sont pas bien suivies, ce d'autant plus que ces relais bénéficient d'un appui relativement faible en terme de renforcement des capacités, de formation et de valorisation de leur travail. Enfin, la stratégie nécessite la mobilisation de moyens financiers énormes, ce qui ne garantie pas sa pérennité.

\section{Le téléphone vert}

Le téléphone a été un moyen au début des interventions utilisé par la population pour manifester son mécontentement et s'opposer aux efforts de lutte contre la pratique. Le CNLPE a jugé opportun d'utiliser ce même canal pour dénoncer les cas éventuels ou avérés d'excision. C'est ainsi que le téléphone «S.O.S Excision » a été mis en place et est pris entièrement en charge par le budget de l'Etat. Aujourd'hui, le CNLPE est doté d'un téléphone vert qui permet à la population de signaler de façon anonyme tout cas à venir d'excision et de dénoncer ceux qui l'ont pratiqué sans frais pour l'appelant. 
Il faut noter que les appels téléphoniques ne sont pas toujours bien archivés. La réponse rapide aux cas de dénonciation nécessite des moyens qui ne sont pas toujours disponibles.

\section{L'arrêt des personnes pratiquant l'excision et leur condamnation}

Sur les données disponibles dans les trois juridictions visitées ${ }^{4}$, plusieurs personnes ont été condamnées. Sur 94 personnes condamnées, 27 ont eu une peine de moins de 6 mois tandis que 3 ont eu un dossier classé sans suite.

\section{Tableau 5: Condamnations au niveau de trois tribunaux}

\begin{tabular}{|l|c|c|c|c|c|c|c|}
\hline & & & \multicolumn{5}{|c|}{ Condamnations } \\
\hline $\begin{array}{l}\text { Tribunal de } \\
\text { Grande } \\
\text { Instance (TGI) }\end{array}$ & Période & $\begin{array}{l}\text { Nombre } \\
\text { de pers }\end{array}$ & $\begin{array}{r} \\
\text { (6 mois }\end{array}$ & $\begin{array}{l}\text { 6 mois } \\
-1 \text { an }\end{array}$ & 1 an + & $\begin{array}{l}\text { Données } \\
\text { manquantes }\end{array}$ & CSS \\
\hline Ouahigouya & $2004-2005$ & $\mathbf{2 1}$ & $\mathbf{1 8}$ & $\mathbf{0}$ & $\mathbf{0}$ & $\mathbf{0}$ & $\mathbf{3}$ \\
\hline Ouaga & $2000-2003$ & $\mathbf{1 7}$ & $\mathbf{6}$ & $\mathbf{1}$ & $\mathbf{4}$ & $\mathbf{6}$ & $\mathbf{0}$ \\
\hline Bobo & $1997-2005$ & $\mathbf{5 6}$ & $\mathbf{3}$ & $\mathbf{1 0}$ & $\mathbf{5}$ & $\mathbf{3 8}$ & $\mathbf{0}$ \\
\hline Total & $1997-2005$ & $\mathbf{9 4}$ & $\mathbf{2 7}$ & $\mathbf{1 1}$ & $\mathbf{9}$ & $\mathbf{4 4}$ & $\mathbf{3}$ \\
\hline
\end{tabular}

\section{L'application variable de la loi}

Bien que de moins en moins de cas signalés échappent à la justice, plusieurs condamnations ont été inférieures aux 3 mois stipulés dans la loi ou sont des condamnations avec sursis. On note également de nombreuses interventions (personnes influentes, proches parents, ...) pour soit éviter la condamnation, soit faire condamner avec sursis ou soit faire libérer les exciseuses, les parents et/ou les complices. Dans certains cas, le dossier est classé sans suite tandis que dans d'autres situations la libération intervient avant l'épuration de la peine. Toutes ces actions ne sont pas de nature à rassurer ceux qui ont décidé de respecter la loi.

\section{Les patrouilles de sensibilisation et de dissuasion}

Les Brigades de Gendarmerie ont opté pour la mise en place de patrouilles de sensibilisation et de dissuasion afin de contribuer à l'élimination progressive de la pratique de l'excision. Ces patrouilles sont réalisées par vingt deux (22) brigades de gendarmerie à travers seize (16) provinces les plus touchées par la pratique de l'excision et couvrent généralement 04 mois (octobre à mai) par année.

Il s'est instauré une meilleure communication entre les gendarmes et les populations qui ne sortent pas seulement pour réprimer mais aussi pour éduquer et sensibiliser. Le recensement, l'identification des exciseuses permet d'assurer une surveillance continue et de dissuader de pratiquer l'excision.

\footnotetext{
${ }^{4}$ Les tribunaux de grande instance de Ouagadougou, Bobo-Dioulasso et Ouahigouya
} 
Cependant les patrouilles ne sont pas réalisées sur tout le territoire national. Là où elles ont lieu, la période ne correspond pas toujours à celle de la recrudescence de la pratique et elles touchent faiblement la population féminine, notamment dans le nord musulman. Ces patrouilles peuvent créer un déplacement transfrontalier de l'excision et donc la clandestinité de la pratique. L'essentiel du financement étant assuré par l'UNICEF, la pérennité de la stratégie n'est pas garantie.

\section{La réparation des séquelles d'excision}

Pour pallier aux problèmes sanitaires des femmes et filles qui souffrent des séquelles de l'excision, le comité avec ses partenaires techniques et financiers ont initié et mis en œuvre la stratégie de réparation des cas de séquelles. Les médecins formés sont chargés de réparer tous les cas de complications liées à l'excision.

Cette stratégie a permis une meilleure visibilité et acceptabilité des activités de lutte contre l'excision et la création d'un environnement favorable à la déclaration des cas de séquelles d'excision. Elle permet aux filles et femmes victimes, une prise en charge médicale gratuite ou à un coût très réduit ${ }^{5}$. Les témoignages de ces filles et femmes souffrants de séquelles permettent de renforcer la sensibilisation.

Cependant ces structures médicales ne sont pas disponibles partout. Les prestations se pratiquent uniquement dans les formations sanitaires avec antenne chirurgicale. On note également le manque de prise en charge psychologique, notamment pour celles qui souffrent dans leur foyer.

\section{Autres types d'intervention par les ONGs et associations}

Parmi les ONG et associations apportant un appui direct, seules quelques unes sont présentées à cause de l'ampleur et de la spécificité de leurs approches.

\section{L'approche holistique par l'éducation aux droits humains et à la santé}

C'est une approche globale qui s'intéresse à plusieurs aspects du développement aussi bien humain que socio-économique. L'excision n'est qu'un thème parmi plusieurs autres thèmes de droit, d'hygiène et de santé.

Il y a une prise de conscience locale accrue des dangers de l'excision et l'identification de l'excision comme une violence faite aux femmes, ce qui a entrainé parallèlement des cas de dénonciation de mariage forcé et de discrimination dans l'éducation des enfants. La déclaration publique d'abandon de la pratique de l'excision devant des autorités coutumières et religieuses reflète l'engagement de la population à abandonner l'excision. Des initiatives sont nées suite à ce programme (construction de case de santé, réparation de points d'eau,...), la recherche d'activités génératrices de revenu (confection de foyer amélioré, ...), l'enregistrement d'actes civiques (établissement de jugements supplétifs d'acte de naissance, de carte d'identité et de livret de famille, cérémonie officielle de mariage civil public des couples) ainsi que l'amélioration de l'hygiène du village.

\footnotetext{
${ }^{5}$ Il est demandé une participation de la patiente qui est constituée de l'achat d'une paire de gants pour l'opération.
} 
Il faut noter le manque de suivi de la déclaration publique d'abandon de la pratique et le manque de mécanisme de consolidation des acquis et de soutien du processus de changement social. Les modules de formation sont essentiellement centrés sur les femmes alors que les hommes sont identifiés comme les décideurs clés. L'absence de système d'autopromotion et de stabilité des formateurs n'ont pas permis de pérenniser les formations au sein des communautés.

\section{L'approche des Imams et des exciseuses}

Cette approche a été mise en œuvre dans la province du Zoundwéogo et avait pour public cible, l'ensemble des chefs religieux musulmans qui devaient mener des activités de sensibilisation dans les mosquées et les exciseuses pour une implication dans la sensibilisation.

Il y a eu une réelle implication des chefs religieux et des chefs traditionnels avec un engagement des populations. Cela a favorisé la sensibilisation dans les mosquées, mais surtout de faire une clarification sur l'origine et le lien entre l'excision et les obligations religieuses. Ces sensibilisations ont permis une large diffusion des messages et de démystifier l'excision aux yeux des fidèles. La ferme conviction de certains leaders a permis de corriger certaines croyances. L'action des exciseuses a encouragé d'autres exciseuses à s'engager dans le processus.

Cependant il faut noter la durée courte de l'intervention et la faible motivation de certains responsables administratifs à continuer ce processus.

\section{L'approche de la Pair Education}

Cette approche a été expérimentée dans la province du Kénédougou avec une association de jeunes (APJAD). Elle suscite l'adhésion et la participation des jeunes dans la sensibilisation des autres jeunes à travers des équipes d'animation permanente appelées « clubs anti-MGF ».

La sensibilisation des jeunes et des adultes est désormais possible dans un environnement traditionnellement hostile aux questions liées à l'excision. Des petites filles non excisées ont été dénommées «filles APJAD » et sont désormais suivies par l'association.

Cet impact est resté limité à deux départements de la province, ce qui ne permet pas de tirer des conclusions généralisables. De plus, il est difficile de mesurer les effets dans cette zone où l'excision se pratique à un âge beaucoup plus élevé.

\section{L'approche des leaders communautaires}

Elle est mise en œuvre par l'association AMMIE (Appui Moral, Matériel et Intellectuel à l'Enfant) dans la province du Yatenga, proche du Mali et reconnue comme la plus touchée par la pratique de l'excision. Il s'est agit de former ces leaders communautaires comme animateurs relais dans cinq départements.

L'engagement d'un député, membre du parlement de la CEDEAO est important. Cela a facilité l'implication et le renforcement des compétences des leaders communautaires, notamment les femmes des villages et des animateurs et animatrices de l'association. Il y a aussi la mise en place de relais communautaires et de comité villageois de vigilance et de surveillance pour 
dénoncer les intentions d'excision. La déclaration publique d'abandon de la pratique de l'excision qui a eu lieu traduit l'engagement des populations à changer de comportement.

L'absence d'évaluation des activités, notamment sur la fonctionnalité des relais et comités villageois de surveillance ainsi que l'absence de suivi de la déclaration publique ne permettent pas de mesurer le changement réel de comportement au sein des populations, surtout parmi les leaders.

\section{L'intégration des modules dans le cursus scolaire}

La Direction de l'Education en Matière de Population du Ministère de l'Education a intégré des modules sur l'excision dans les curricula.

Les élèves dans leur grande majorité sont plus informés sur les questions relatives à l'excision. Il existe un environnement de discussion sur l'excision à travers l'association des parents d'élèves mais également à la maison entre parents et enfants. La signature de la lettre circulaire conjointe des deux ministères chargés de l'enseignement permet de généraliser l'introduction des modules dans les programmes scolaires. Aussi, l'engagement politique se manifeste encore une fois avec l'intégration des modules dans le plan de développement de l'éducation de base (PDDEB) et l'actuelle extension dans quelques écoles primaires et secondaires de deux régions du pays (l'Est et Sud-ouest).

L'insuffisance d'équipement des écoles primaires en zone rurale où l'électricité n'est pas disponible ne permet pas toujours la projection de films et diapositives sur la question. La généralisation de l'intégration des modules n'étant pas encore effective dans toutes les directions régionales de l'enseignement, certains élèves ne pourront pas bénéficier de ces modules dans un délai court. L'absence de ces modules dans le programme de formation professionnelle des instituteurs et des enseignants du secondaire ne permet pas de former directement des enseignants capables de dispenser ces modules.

\section{L'approche des communicateurs traditionnels}

La particularité de cette stratégie de sensibilisation de masse réside dans l'utilisation des communicateurs traditionnels communément appelés griots ou griottes. Le département de Bobo-Dioulasso (Chef lieu de la province du Houet) a été choisi pour cette approche vue l'importance et la place de ces communicateurs traditionnels dans la vie des communautés.

Une sensibilisation grand public qui mobilise beaucoup de monde, notamment pendant les occasions de réjouissances ainsi que les animations en langues locales permettent une large compréhension des messages.

La sensibilisation de masse reste diffuse tandis que l'absence d'évaluation ne permet pas de tirer des conclusions sur les effets réels de la stratégie.

\section{La radio locale avec les femmes leaders}

Cette approche a mis l'accent sur l'utilisation de la radio locale dans la ville de Banfora, notamment celle d'une association de femmes, pour des campagnes de sensibilisation avec des 
femmes leaders. Sa particularité réside dans l'implication des femmes dans la lutte contre la pratique, à travers une radio locale.

La mobilisation des femmes a été remarquable, ce qui a entrainé l'adhésion de leurs conjoints. Les activités ont permis d'augmenter le niveau de connaissance des femmes, parce que les émissions réalisées par les femmes sont beaucoup plus écoutées. On note également une prise de conscience des femmes par rapport à la question de l'excision et les animations en langues locales permettent la compréhension des messages.

La sensibilisation reste toujours une sensibilisation de masse et la focalisation sur les femmes réduit quelque peu l'impact sur la prise de conscience des autres hommes (n'ayant pas de femme ou dans l'association) sur le fait qu'il ne s'agit pas uniquement de problème de femmes.

\section{L'intégration dans le projet1000 jeunes filles}

Ce programme à l'intention des adolescentes rurales intitulé Projet des centres de production et de formation pour jeunes filles $(\mathrm{CPF} / \mathrm{JF})$ est communément appelé projet 1000 jeunes filles ${ }^{6}$. Ce projet est issu de la mise en œuvre des engagements pris en 1994 par le chef de l'Etat dans le cadre de l'amélioration des conditions de vie des femmes et de la population en général. Il vise particulièrement des adolescentes de 14 à 18 ans des 45 provinces du pays. La question de l'excision a été intégrée dans le programme de formation

Une intégration des questions de l'excision dans l'ensemble des activités liées à la santé de la reproduction a permis une forte adhésion des filles. Une évaluation ultérieure montre qu'aucune des filles sorties du centre de formation n'a pratiqué l'excision sur ses enfants de sexe féminin.

Le manque de suivi des filles sorties du centre ne permet pas de mesurer leurs capacités à résister aux pressions.

\section{L'intégration de l'excision dans le projet de renforcement des capacités des adolescentes}

Ce projet intervient principalement en milieu rural, dans deux districts sanitaires géographiquement opposés (Kombissiri et Fada). Le projet vise à renforcer les «compétences de vie » et les droits au développement et à la santé y compris l'excision, à rendre disponibles et accessibles les services de santé sexuelle et reproductive et à améliorer la qualité de vie de ces adolescentes (mariées ou non mariées) âgées de 10 à 19 ans.

Des services sociaux et sanitaires sont renforcés en compétence et en matériel, et sont réhabilités pour assurer des services de qualité, notamment la prise en charge des accouchements. Aussi, il y a une meilleure information sur l'excision puisque des femmes paires éducatrices assurent l'éducation de porte à porte.

Il y a un faible contrôle du choix des paires éducatrices qui peuvent ne pas être toutes convaincues de la nécessité de l'abandon de l'excision.

\footnotetext{
${ }^{6}$ Le centre accueille 1000 jeunes filles envoyées par toutes les provinces.
} 


\section{Appui à la Campagne de lutte contre la pratique des mutilations génitales féminines dans les régions du Nord et de l'Est}

L'ONG Médicus Mundi Andalucia intervient dans deux régions du burkina. En plus de l'appui institutionnel, l'appui aux activités des radios locales et la formation des agents de santé en technique de réparation des séquelles de l'excision, l'ONG utilise surtout la stratégie d'implication des exciseuses dans les campagnes de sensibilisation en les identifiant et en leur apportant la formation nécessaire.

On note une plus grande mobilisation autour de la question de l'excision. Deux cérémonies publiques de dépôt de couteau des exciseuses ont eu lieu à Fada et Ouahigouya. Il faut noter un manque de suivi des exciseuses après les déclarations publiques afin de maintenir l'engagement pris durant les déclarations de dépôt des couteaux mais aussi l'absence dévaluation de ces activités dans le changement de comportement de la communauté.

\section{Conclusion}

Toutes ses approches, stratégies et actions ont pour objectif d'influencer fortement le niveau de connaissance et le comportement des populations. Grâce au plaidoyer et à la formation, les groupes sociaux et les groupes religieux ont été sensibilisés, ce qui a permit de susciter un engagement des leaders par la mise en place de comités des chefs traditionnels, de comités de soutien des associations islamiques (1996) et des chefs coutumiers (1997).

Les formations des députés (pour l'adoption de textes règlementaires), des agents de santé (pour reconnaître et réparer les séquelles de l'excision), des magistrats (pour une application rigoureuse de la loi), des forces de sécurité (pour la sensibilisation et les interventions sur le terrain) sont autant d'action qui ont permis au CNLPE et a ses partenaires d'avoir des interventions structures et holistique par la variété des canaux de diffusion.

Le lobbying que mène le CNLPE a permis une adhésion effective des partenaires au développement. Cette adhésion se traduit en tout temps par des actions et des appuis multiformes qui permettent la mise en œuvre des activités. Ces appuis ont permis au comité de disposer d'un site web pour une plus grande visibilité et d'un centre de documentation équipé sur les questions de la SR, spécialement les questions relatives aux femmes et aux filles.

La campagne continue a eu pour effet de rendre moins tabou la question de l'excision. Si au départ les agents de terrain étaient parfois agressés physiquement et les femmes traitées de femmes faciles, dévergondées et/ou de prostituées, le climat est aujourd'hui plus accueillant et moins agressif. Aussi bien dans la presse publique que privée (écrite et audio-visuelle), le sujet de l'excision est désormais traité sans complaisance (utilisation de mannequins de femmes nues, d'images et de photos choquantes) et les photos des excisées, exciseuses et complices ainsi que de parents sont parfois présentées. Les média se font le relais systématique de ces images et photos choquantes. Ces différentes actions de sensibilisation ont permis une amélioration remarquable du niveau de connaissance des populations sur la question de l'excision, notamment son aspect interdiction.

Malgré des difficultés de financement, d'absence d'indépendance dans le choix des stratégies, de mobilité du personnel, d'application variable de la loi, le Burkina fait preuve de beaucoup de 
détermination pour que la pratique de l'excision soit abandonnée. L'effort de coordination ou l'assistance aux différents intervenants mérite aussi d'être souligné.

\section{L'évolution de la pratique de l'excision}

La majeure partie des études sur l'excision utilisent les données recueillies auprès des femmes de 15-49 ans, même lorsqu'il s'agit des données sur la prévalence de l'excision de leurs filles de moins de 15 ans. La première enquête quantitative nationale date de 1996, peu avant l'adoption de la loi. Depuis, les activités de sensibilisation se sont intensifiées sur l'ensemble du territoire. Les filles nées à partir de cette date ont actuellement moins de 10 ans. Cette tranche d'âge permet de mieux cerner l'effet de la loi et des interventions sur la pratique de l'excision. Comme déjà précisé dans la méthodologie, les données présentées dans cette section proviennent de plusieurs enquêtes. Elles n'ont pas pour but d'effectuer une comparaison car n'utilisant pas exactement les mêmes méthodes, cependant elles portent toutes sur un échantillon représentatif des femmes âgées de 15-49 ans.

\section{Evolution de la prévalence de l'excision}

\section{Prévalence de l'excision chez les filles et les femmes}

Bien que la majeure partie des études existantes sur l'excision ne couvrent pas l'ensemble du territoire, on note néanmoins une prévalence beaucoup plus faible chez les filles de $0-10$ ans, comparativement aux filles et femmes plus âgées. L'écart entre les filles et leur mère est de plus de $50 \%$ et cet écart a tendance à augmenter au fil du temps. Depuis 1996, la prévalence chez ces filles reste inférieure à $25 \%$. Cependant, force est de constater que depuis 2002, la tendance semble se maintenir autour de $20 \%$.

\section{Graphique 4 : Evolution de la pratique de l'excision depuis 1996}

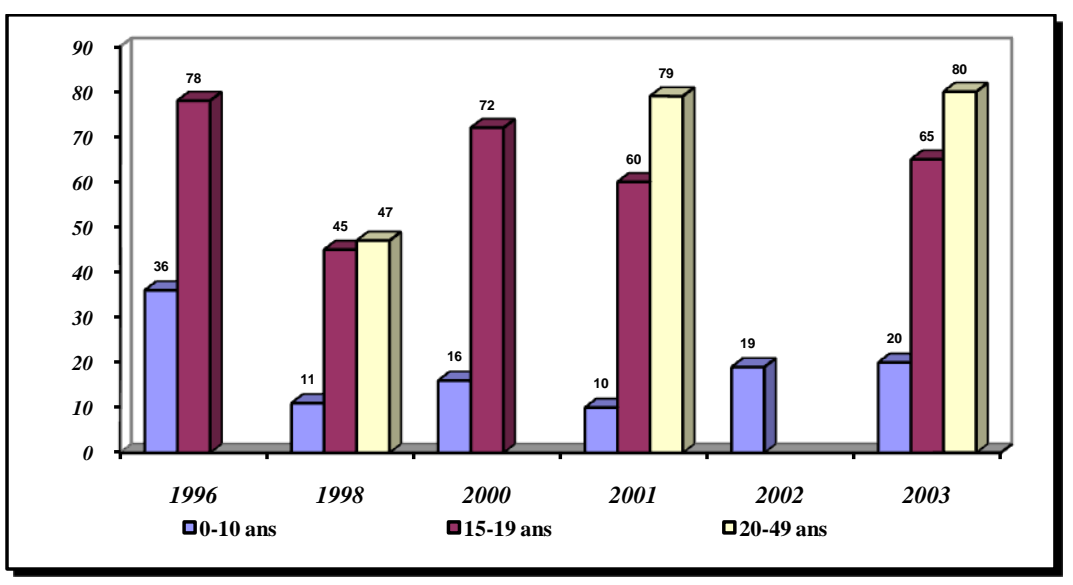

\section{L'évolution selon l'âge de la mère}

En analysant l'évolution de l'excision parmi les petites filles suivant l'âge des mères, on notera que les jeunes femmes (15-24 ans) ont moins de filles excisées comparativement aux femmes plus âgées. Cette tendance est restée la même entre 1999 et $2003^{7}$.

\footnotetext{
${ }^{7}$ EDS 1999 et EDS 2003
} 
Tableau 6: Prévalence de l'excision des filles au fil du temps suivant l'âge de la mère, selon que la mère a une fille en vie

\section{Evolution de l'âge à l'excision}

\begin{tabular}{lccccccc}
\hline \multicolumn{9}{c}{ Age de la mère } \\
& $15-19$ & $20-24$ & $25-29$ & $30-34$ & $35-49$ & Tot. & Eff. mère \\
\hline EDS 1999 & $8,5(118)$ & $14,8(494)$ & $29,3(706)$ & $45,9(639)$ & $65,4(1541)$ & 45,5 & 3499 \\
EDS 2003 & $5,7(229)$ & $9,4(1029)$ & $17,4(1503)$ & $28,6(1271)$ & $49,5(3325)$ & 32,4 & 7356 \\
\hline
\end{tabular}

L'âge à l'excision illustre la signification sociale accordée à la pratique, notamment en ce qui concerne les cérémonies et rites. Le tableau ci-dessous compare l'âge à l'excision des mères et des filles en 1999 et en 2003. Entre les deux périodes, la proportion de filles excisées entre 5 et 10 ans a sensiblement diminué $(\mathrm{p}<0,01)$ au profit des filles de moins de 5 ans. Elle passe de $54 \%$ à $61 \%(\mathrm{p}<0,01)$, soit une augmentation de $13 \%$.

Tableau 7: Répartition des filles et des mères par âge à l'excision, selon les deux EDS

\begin{tabular}{|c|c|c|c|c|c|c|c|c|c|c|c|c|c|}
\hline & \multicolumn{10}{|c|}{ Age à l'excision des mères et des filles } & \multirow{3}{*}{ Total } & \multirow{2}{*}{\multicolumn{2}{|c|}{ Effectif }} \\
\hline & 0 - & & $5-$ & & $11-$ & & $15+$ & & $\mathrm{NS}$ & & & & \\
\hline & Mères & Filles & Mères & Filles & Mères & Filles & Mères & Filles & Mères & Filles & & Mères & Filles \\
\hline EDS 99 & 24,4 & 53,8 & 40,3 & 39,6 & 4,3 & 2,0 & 2,2 & 0,7 & 28,9 & 3,8 & 100 & 4615 & 1591 \\
\hline EDS 2003 & 50,7 & 61,1 & 34,0 & 32,3 & 4,0 & 1,5 & 2,1 & 0,4 & 9,1 & 4,6 & 100 & 9552 & 2380 \\
\hline
\end{tabular}

La peur de la loi et le désir de continuer à exciser les filles conduiraient donc les parents à exciser les filles avant 5 ans, permettant non seulement de réduire les possibilités de refus de la part des filles mais également parce que l'excision des petites filles n'attire pas l'attention et peut se faire de façon clandestine. De plus, pour les communautés la fille ne gardera pas le souvenir douloureux de l'opération. L'âge moyen pour les filles récemment excisées est de 4,12 ans, avec la majeure partie des filles excisées avant un an. Selon l'étude réalisée par Sangli en 2001 dans sept provinces, trois provinces (Ganzourgou, Gnagna et Kouritenga) avaient un âge moyen à l'excision inférieur à 5 ans. Dans le Soum, l'âge moyen y était de 1 an. 


\section{Les disparités régionales ethniques et culturelles de la pratique de l'excision}

Plusieurs études et recherches ont montré que la prévalence de l'excision variait selon le groupe ethnique d'appartenance.

Tableau 8: Prévalence de l'excision par groupe d'âges et selon l'appartenance ethnique (EDS 98 et 98/99)

\begin{tabular}{l|cc|cc|cc|}
\hline Groupes ethniques & \multicolumn{2}{|c|}{$\mathbf{2 0 - 4 9}$ ans } & \multicolumn{2}{c|}{ 15-19 ans } & \multicolumn{2}{c|}{$\mathbf{0 - 1 0 \text { ans } ^ { 8 }}$} \\
\cline { 2 - 7 } & $\mathbf{1 9 9 8 / 9 9}$ & $\mathbf{2 0 0 3}$ & $\mathbf{1 9 9 8 / 9 9}$ & $\mathbf{2 0 0 3}$ & $\mathbf{1 9 9 8 / 9 9}$ & $\mathbf{2 0 0 3}$ \\
\hline \multirow{2}{*}{ 1. Mossi } & 76,5 & 82,5 & 66,3 & 68,6 & $24,3(3318)$ & $19,2^{*}$ \\
& $(3101)$ & $(5885)$ & $(911)$ & $(1753)$ & & $(5800)$ \\
2. Goin-Sénoufo & 91,6 & 92,8 & 83,3 & 68,9 & $27,0(115)$ & 25,6 \\
& $(119)$ & $(361)$ & $(29)$ & $(114)$ & & $(375)$ \\
3. Lobi-Dagara & 90,8 & 72,0 & 79,1 & 57 & $33,3(114)$ & $17,2^{*}$ \\
& $(119)$ & $(522)$ & $(43)$ & $(124)$ & & $(512)$ \\
4. Peul & 68,5 & 72,3 & 59,3 & 57,4 & $30,5(364)$ & $22,6^{*}$ \\
& $(357)$ & $(728)$ & $(86)$ & $(197)$ & & $(725)$ \\
5. Gourmantché & 50,5 & 69,8 & 37,8 & 62,2 & $15,9(522)$ & $11,6^{* *}$ \\
& $(416)$ & $(702)$ & $(111)$ & $(156)$ & & $(760)$ \\
6. Gourounsi & 48,1 & 53,1 & 39,1 & 32,0 & $10,9(274)$ & 6,9 \\
& $(271)$ & $(320)$ & $(70)$ & $(99)$ & & $(289)$ \\
7. Bobo & 89,2 & 89,4 & 79,5 & 76,5 & $43,1(318)$ & $31,4^{*}$ \\
& $(324)$ & $(781)$ & $(113)$ & $(243)$ & & $(754)$ \\
8. Samo & 90,5 & - & 75 & - & 48,0 & - \\
& $(105)$ & & $(36)$ & $-198)$ & $(96)$ \\
Ens. pays & 73,7 & 79,9 & 64,2 & 65,0 & $25,0(5312)$ & $19,8^{*}$ \\
& $(5001)$ & $(9701)$ & $(1444)$ & $(2776)$ & $(9689)$ \\
\hline
\end{tabular}

Test de signification: $*=0,01 ; * *=0,005$

${ }^{8}$ Les données sur l'excision des filles n'ont pas le même groupe cible selon les deux EDS. En 1999, la question était relative aux filles aînés tandis qu'en 2003, la question s'adresse aux filles les plus récemment excisées, ce qui fondamentalement va différer des filles aînées. 


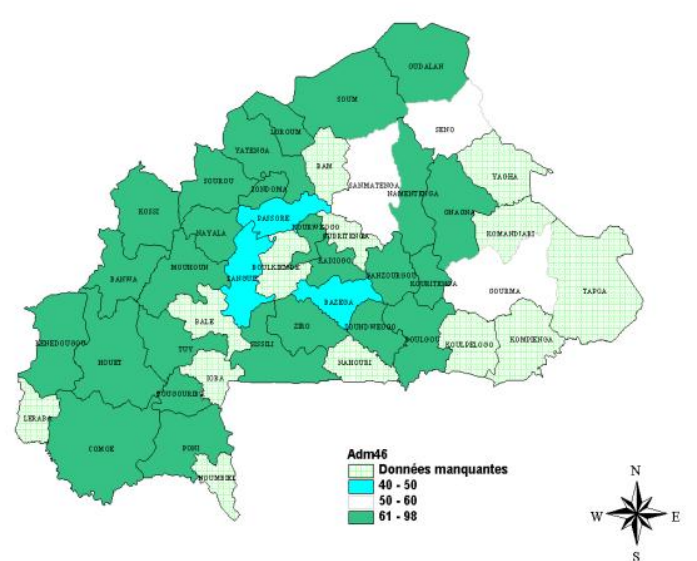

Au Burkina, la diversité culturelle (une soixantaine d'ethnies) conduit très souvent à des regroupements dont les critères sont de trois ordres: suivant l'ancienneté de l'installation (autochtones et autres), suivant la nature centralisée ou non du système politique, suivant la langue ou suivant la combinaison de ces différents critères (langue, système politique, ancienneté, ...). Le critère linguistique est retenu parce qu'il s'apparente à la division administrative du pays en provinces.

Chez les filles de moins de 10 ans, on note partout une baisse significative entre 1999 et 2003, sauf chez les filles goins-sénoufo où la tendance est restée constante. Les prévalences les plus élevées au sein de ce groupe d'âge se rencontrent par ordre décroissant chez les bobo, les goins-sénoufo, les mossi, les peuls, les gourmantché et les gourounsi. La correspondance par province ou région donnerait respectivement les Hauts Bassins, le Sud-ouest, le centre en général, le Nord et le Sahel, l’Est et le centre sud.

\section{Les facteurs socio-démographiques liés à la pratique de l'excision}

Selon le milieu de résidence

L'excision baisse plus rapidement en milieu urbain qu'en milieu rural. En considérant la seule ville de Ouagadougou, la capitale politique, le niveau de prévalence est en baisse significative ( $\mathrm{p}<0.01)$ entre 1999 (79\%) et 2003 (73\%). Le milieu rural contribue pour beaucoup dans l'augmentation du niveau observé sur le plan national puisqu'il observe une augmentation significative ( $\mathrm{p}<0.01)$ durant la même période (EDS 1999;2003).

\section{Selon le niveau d'instruction de la mère}

Au Burkina, l'instruction est fortement associée $(\mathrm{p}<0,05)$ à un niveau faible de prévalence. C'està-dire que les femmes instruites ont moins de filles excisées (voir tableau ).

Tableau 9 : Prévalence de l'excision des filles suivant le niveau d'instruction de la mère, selon que la mère a une fille en vie (EDS 98/99 et 2003)

\begin{tabular}{lccccc}
\hline & \multicolumn{3}{c}{ Niveau d'instruction de la mère } & & \\
\cline { 2 - 4 } & $\begin{array}{c}\text { Sans } \\
\text { instruction }\end{array}$ & Primaire & Secondaire et + & Total & Eff. mères \\
\hline EDS 1999 & $47,3(3151)$ & $35,1(239)$ & $16,4(110)$ & 45,5 & 3499 \\
EDS 2003 & $34,2(6447)$ & $24,0(588)$ & $9,9(323)$ & 32,4 & 7356 \\
\hline
\end{tabular}


Selon la religion de la mère

Les données nous montrent que la prévalence de l'excision est plus élevée chez les femmes musulmanes $(p<0,05)$. Ceci est vrai pour les mères et pour les filles quel que soit le groupe d'âge des mères (tableau ).

Tableau 10 : Prévalence de l'excision des filles au fil du temps suivant la
religion de la mère, selon que la mère a une fille en vie (EDS 1999 et 2003)
\begin{tabular}{ccccccc}
\multicolumn{5}{c}{ Religion de la mère } & & \\
\cline { 2 - 4 } & Chrétiennes & Musulmanes & Autres & Total & Eff. mères \\
\hline EDS 1999 & $36,5(931)$ & $49,1(2009)$ & $42,2(559)$ & 45,5 & 3499 \\
EDS 2003 & $22,5(1927)$ & $37,0(4472)$ & $30,7(957)$ & 32,4 & 7356 \\
\hline
\end{tabular}

A la recherche des facteurs de risque d'excision pour les filles

Pour déterminer les facteurs de risque d'excision pour les filles, une analyse multivariée a été menée. Pour tenir compte de l'évolution dans le temps, les données répétées ont été considérées, en l'occurrence les données de l'EDS. Les principales variables indépendantes sont: l'âge, la situation matrimoniale, le niveau d'instruction, la religion, l'ethnie. L'utilisation de la contraception moderne et le type d'emploi sont deux variables servant à approcher le phénomène de la modernité.

En 1999, le niveau d'instruction, la religion et l'ethnie apparaissent comme des facteurs différenciant les filles des femmes. La relation entre l'excision et le niveau d'instruction disparaît, une fois confrontée aux autres variables, de sorte que seules les filles des femmes de niveau secondaire se distinguent nettement avec un risque plus faible. Quant à la religion, les filles de femmes musulmanes continuent de courir plus de risque d'être excisées, comparées aux filles de femmes des autres religions.

Les principaux groupes ethniques d'appartenance des filles où le risque d'être excisé est grand sont : les peuls, les Bobo, et les Samo. Les filles des femmes de ces groupes ethniques ont entre 2,4 à près de 7 fois plus de chance d'être excisées, comparativement aux filles des femmes mossi, Goins-Sénoufo, Lobi-Dagara et Gourmantché. A l'inverse, appartenir au groupe ethnique Gourounsi donne plus de chance d'échapper à l'excision.

En 2003, la relation entre la situation matrimoniale des femmes et l'excision des filles est demeurée non significative. Le milieu de résidence et le statut d'employé apparaissent désormais comme des facteurs de risque de l'excision pour les filles. La fille qui réside avec sa mère en milieu rural coure plus de risque d'être excisée, comparée à celle qui vit en zone urbaine. Quant au niveau d'instruction, il demeure toujours un facteur de moindre risque pour les filles, surtout lorsque la mère a au moins le niveau secondaire. 
Tableau 11 : Odds ratio du modèle de régression logistique sur l'excision des filles selon les données de l'EDS II (1998/99) et l'EDS III (2003)

\begin{tabular}{|c|c|c|}
\hline Caractéristiques & EDS 1998/99 & $\begin{array}{l}\text { EDS } \\
2003\end{array}$ \\
\hline Age & $1,392^{* \star *}$ & $1,404^{* * *}$ \\
\hline $\mathrm{Age}^{2}$ & $0,997^{* * *}$ & $0,997^{* * *}$ \\
\hline \multicolumn{3}{|l|}{ Milieu de résidence } \\
\hline Urbain (r) & 1,000 & 1,000 \\
\hline Rural & 1,005 & $1,248^{\star *}$ \\
\hline \multicolumn{3}{|l|}{ Niveau d'instruction } \\
\hline Sans instruction $(r)$ & 1,000 & 1,000 \\
\hline Primaire & 0,859 & 0,873 \\
\hline Second + & $0,226^{* \star *}$ & $0,349^{* * *}$ \\
\hline \multicolumn{3}{|l|}{ Religion } \\
\hline Musulmans (r) & 1,000 & 1,000 \\
\hline Chrétiens & $0,614^{* * *}$ & $0,490^{* * *}$ \\
\hline Autres & $0,790^{* *}$ & $0,699^{\star * *}$ \\
\hline \multicolumn{3}{|l|}{ Situation matrimoniale } \\
\hline Célibataire (r) & 1,000 & 1,000 \\
\hline Monogame & 3,416 & 1,346 \\
\hline Polygame & 3,443 & 1,289 \\
\hline Divorcé/veuve/séparée & 2,137 & 1,613 \\
\hline \multicolumn{3}{|l|}{ Ethnie } \\
\hline Mossi (r) & 1,000 & 1,000 \\
\hline Goin-Sénoufo & 1,135 & $1,560^{* * *}$ \\
\hline Lobi-Dagara & 1,331 & $0,746^{\star *}$ \\
\hline Peul & $2,401^{* * *}$ & $1,499^{* * *}$ \\
\hline Gourmantché & 0,971 & $0,618^{* * *}$ \\
\hline Gourounsi & $0,360^{* * *}$ & $0,235^{\star * *}$ \\
\hline Bobo & $4,325^{\star \star *}$ & $2,014^{* * *}$ \\
\hline Samo & $6,620^{* * *}$ & - \\
\hline Utilise la contraception moderne & 1,009 & 1,072 \\
\hline Employég & 0,730 & $0,361^{* *}$ \\
\hline$N$ & 3456 & 7358 \\
\hline
\end{tabular}

En ce qui concerne la religion, la tendance reste la même avec les données de 1999, puisque le fait d'être de la religion musulmane augmente le risque d'avoir une fille excisée, comparativement aux autres. Quant à l'appartenance ethnique, les filles qui courent le plus grand risque d'être excisées sont dans l'ordre d'importance du groupe bobo, goin-sénoufo et peuls. En ce qui concerne l'activité économique, il apparaît que le fait d'avoir une mère fonctionnaire ou employé de bureau fait courir moins de risque à la fille d'être excisée.

\footnotetext{
${ }^{9}$ Employées de bureau et fonctionnaires
} 


\section{Evolution des attitudes}

L'approbation de la pratique a significativement évolué depuis 1996, même si les niveaux diffèrent selon les zones d'enquête (tableau 12). Aujourd'hui, moins de femmes et d'hommes approuvent encore l'excision. La proportion de femmes et des hommes approuvant la pratique a diminué de moitié ( $\mathrm{p}<0.01)$ entre 1996 et 2003. L'approbation de la pratique concerne environ $10 \%$ des femmes et des hommes, sauf dans la zone LSC où seulement $3 \%$ des femmes approuvent la pratique. Dans certains lieux, les hommes représentent 25\% (Sangli, 2001).

\begin{tabular}{|c|c|c|c|c|}
\hline \multirow[b]{2}{*}{ Etudes } & \multicolumn{2}{|c|}{ Approbation de l'excision } & \multicolumn{2}{|c|}{$\begin{array}{c}\text { Intentions } \\
\text { d'exciser les futures filles }\end{array}$} \\
\hline & Femmes & hommes & femmes & hommes \\
\hline INSD (1996) & $47,1(1654)$ & $38,3(397)$ & - & - \\
\hline $\begin{array}{l}\text { LSC } 1996 \\
\text { LSC } 1998 \\
\text { EDS } 1998 \\
\text { EDS } 2003\end{array}$ & $\begin{array}{l}50,7(2795) \\
24,7(2605) \\
21(5559) \\
17,1(12060)^{10}\end{array}$ & $\begin{array}{l}41,4(1869) \\
24,1(1716) \\
17(2390) \\
13,2(3366)\end{array}$ & $\begin{array}{l}- \\
16,4(2605) \\
4,3(6445)^{11} \\
5,2(7540)\end{array}$ & $\begin{array}{l}- \\
14,3(1716) \\
- \\
-\end{array}$ \\
\hline $\begin{array}{l}\text { PEBC } 2001 \\
\text { PEBC } 2003\end{array}$ & $\begin{array}{l}11(662) \\
3,1(745)\end{array}$ & $10(697)$ & $\begin{array}{l}3(662) \\
0,4(745)\end{array}$ & $3(697)$ \\
\hline Ouédraogo D.2000 & $10(879)$ & - & - & - \\
\hline Sangli 2001 & $15,4(1632)$ & 24,7 (983) & 4,5 (1632) & $9,1(983)$ \\
\hline Ouédraogo I. 2001 & $13,1(1861)$ & - & - & - \\
\hline Kabore 2002 & $14(3941)$ & - & - & - \\
\hline
\end{tabular}

Quant aux parents désireux d'exciser les futures filles, leur nombre a également diminué, passant de $15 \%$ en 1998 à 5\% en 2003 et même à 1\% dans certaines zones. En 1996, 40\% des hommes interrogés estimaient la pratique de l'excision obligatoire avec comme principales raisons la coutume et la tradition. Dans le même temps, près d'un quart des hommes interrogés avaient exprimé leur préférence pour une femme excisée tandis que 60\% étaient restés indécis sur la question. En 2003, la moitié des femmes a déclaré qu'il n'y a pas d'avantage à exciser les filles.

Pour les femmes favorables à la pratique, la principale raison c'est la coutume et la tradition $(71 \%)$. Les trois principales autres raisons les plus évoquées sont l'hygiène, la préservation de la virginité (éviter l'immoralité) et la nécessité religieuse. Deux raisons essentielles sont évoquées par les femmes défavorables à l'excision : les complications médicales $(51 \%)$ et l'interdiction de la loi $(44 \%)$.

\footnotetext{
${ }^{10}$ La question de l'approbation qui est adressée aux femmes connaissant l'excision est libellée ainsi : pense que l'excision doit être maintenue

${ }^{11}$ L'enquêtée n'a pas sa fille aînée excisée et à l'intention de l'exciser
} 


\section{Conclusion}

Les données présentent un niveau de l'excision toujours élevé chez les femmes en âge de procréer tandis que la tendance est en baisse chez les filles de moins de 10 ans. Cette tendance est accompagnée d'une évolution positive des attitudes. Il y a donc lieu de mesurer l'état actuel des comportements et d'identifier les facteurs explicatifs de l'évolution de la pratique de l'excision au regard du caractère récent de l'environnement légal à la lutte.

\section{Analyse des perceptions et comportements des communautés}

Une enquête qualitative a été menée dans 5 provinces du Burkina afin d'évaluer la situation et identifier les facteurs pouvant expliquer cette baisse, de tenter de comprendre les effets des interventions et de mettre en évidence les perceptions des populations sur quelques programmes d'intervention (voir méthodologie pour plus d'information). La collecte des informations essentiellement qualitatives et leur analyse se base sur la carte mentale et le modèle de diffusion de l'innovation (voir p. ). Un rapport complet des résultats de cette enquête peut être trouvé dans le rapport

\section{Un niveau de connaissance de l'excision élevé}

La connaissance des différents types d'excision et leurs conséquences

Dans les 5 régions de collecte des données, la connaissance des types d'excision est élevée, avec une meilleure connaissance chez les plus jeunes et les plus instruits. Si le lien entre le type d'excision et la gravité des conséquences est aussi connu, il reste que ce sont surtout les conséquences sanitaires qui sont les plus rapportées, notamment les conséquences liées à l'accouchement. Dans une moindre mesure, le lien est établi entre les conséquences sanitaires et les autres types de conséquences telles que les conséquences psychologiques, sociales ou psycho-sexuelles.

"Il y a des excisions où on enlève les grandes lèvres, les petites lèvres, d'autre où on enlève le clitoris simplement. Il y a des excisions avec sutures »(Focus jeunes mixtes, Kadiogo).

\section{Les sources d'information}

Ces sources sont diversifiées, mais les principaux canaux sont la radio et la télévision qui touchent les provinces et les villages les plus reculés.

"Moi j'ai appris dans la télé, les radios, les journaux, des fois aussi on fait les théâtres » (focus adultes mixtes, Oudalan)

Les autres sources sont les théâtres forum, les causeries débat, les leaders d'opinion, les actions éducatives des associations locales, notamment le porte à porte. Les images et les exemples de cas qui sont présentés par les media retiennent surtout l'attention, au détriment du contenu exact des messages qui n'est pas toujours retenu. En effet, les informations sous forme visuelles, films, vidéo, documentaires, mettant en avant la souffrance des personnes excisées marquent plus les esprits que les discours.

"Pour la pratique, c'est dans les causeries. Pour les conséquences de l'excision c'est à la radio Taamba, radio Ouaga, radio nationale »(focus adultes mixtes, Gourma) 


\section{Une régression de la pratique}

\section{Une pratique en baisse}

Dans toutes les zones enquêtées (milieu urbain et milieu rural, hommes et femmes, jeunes et adultes), tous s'accordent à confirmer le fait que la pratique de l'excision est en recul. Un recul admis comme étant à la fois lié à l'évolution de l'environnement socio-éducatif et au caractère répressif de la loi condamnant la pratique.

«Le seul constat est que depuis quelques années, on en parle plus et on ne voit plus les gens exciser leurs enfants. En tout cas pas dans le rayon de ma connaissance » (Focus adultes femmes, Kadiogo)

"Oui, je te dis que tout le monde a abandonné ici à Saouga. S'il y a quelqu'un qui excise tout le monde va savoir, car l'information circule rapidement ici. » (Entretien Leader Religieux musulman, 75 ans, favorable à la pratique de l'excision, Oudalan)

\section{La durée d'abandon}

Bien que les idées divergent quant à la période exacte du début de l'abandon de la pratique, tous (dans les différentes zones d'enquête) s'accordent à dire que le phénomène n'est pas très récent. Le début de l'abandon est situé entre 5 ans et 8 ans. "Je peux dire que ça fait 7 ans que je n'ai pas vu ça dans notre communauté selon moi les hommes, et les femmes ont écouté les conseils et comme ils l'ont écarté. c'est bien. »(Focus adultes hommes, Gourma)

Dans l'Oudalan par exemple, le véritable changement aurait commencé ces cinq dernières années. Chez les populations chrétiennes, l'abandon est plus ancien et plus radical, dû probablement à l'opposition affichée depuis la période coloniale par les missionnaires.

«Ici, chez nous, si ma mémoire est bonne on a commencé à parler de ça il ya environ 7 ans. Mais je suis sûre que c'est depuis six ans maintenant que les gens n'excisent plus. » (Entretien homme, leader religieux, 54 ans, musulman, Ecole coranique, non favorable à la pratique de l'excision, Oudalan)

\section{Les facteurs de modification du comportement}

Beaucoup de personnes ont adopté la nouvelle norme de non excision. Les facteurs explicatifs sont aussi bien endogène que exogène.

\section{L'effet de la répression}

La loi est bien connue par les populations, notamment son caractère répressif. La stratégie de dénonciation mise en place par le CNLPE est également très connue et suscite la peur au sein des populations. Cependant, le contenu plus détaillé de la loi (le montant des amendes, les peines et la classification des catégories de personnes condamnables) n'est pas aussi bien connu.

"Si on t'arrête, on t'enferme et en plus tu dois payer une amende. On dit que l'amende va de $200.000 \mathrm{~F}$ pour la moins chère et la peine d'emprisonnement la plus courte est de 6 mois.» responsable d'association des femmes, Bazèga)

\section{L'effet des sensibilisations}

Pour beaucoup de personnes, les sensibilisations ont eu un effet manifeste et ont beaucoup contribué à changer la vision des populations sur la question. De sorte que le changement est beaucoup plus perceptible au sein des filles jeunes (de moins de 15 ans), correspondant à la durée de mise en place des mesures légales interdisant la pratique. Aussi, parler de l'excision et de la non-excision n'est plus offusquant. 
"C'est progressivement que les mentalités ont changé avec la « sensibilisation». Ils ont vu à la télé certains cas d'excision qui ont posé des problèmes ou bien des exciseuses qu'on a emprisonnées » (focus adultes mixtes, Houét)

"Aujourd'hui avec les sensibilisations, une femme peut se déclarer qu'elle n'est pas excisée, elle a la parole. Dans le temps elle ne pouvait pas parce que les gens trouvaient que c'était mal » (Pasteur, Gourma)

La complexité de la pratique de l'excision

La mise en ouvre de la loi a beaucoup contribué au recul de la pratique. Malgré quelques résistances, le sentiment contraignant et obligatoire de la loi est fortement perceptible par tous et dans toutes les zones. La mise en place des patrouilles de sensibilisation et de dissuasion a été un obstacle à la pratique de l'excision.

"Actuellement, il y a beaucoup de personnes qui disent qu'ils vont exciser leurs enfants mais ils n'ont pas la force de faire. Ils ont peur » (Leader femme, Houet).

"Nous les hommes, nous avions refusé. Nous ne pouvions pas accepter une femme avec un clitoris. Mais lorsqu'on a compris qu'il y a un risque d'emprisonnement, nous nous sommes dit que c'est mieux d'arrêter " (Focus adultes mixtes, Gourma)

"Il y a eu une équipe, venu à Saouga ici avec la gendarmerie. Les gendarmes ont parlé devant tout le monde et expliqué qu'il y a un risque d'emprisonnement» (Leader Religieux, Oudalan)

Ainsi avec tous les dispositifs mis en place, la pratique de l'excision est devenue plus complexe pour les communautés. Le risque d'emprisonnement décourage ceux qui n'ont pas été convaincu par les sensibilisations sur les conséquences de l'excision.

\section{La compatibilité avec les nouvelles valeurs}

L'implication de certains dépositaires de la tradition pour l'abandon de la pratique a reçu l'adhésion des populations. Cela a permis de séparer les justificatifs de type coutumier et traditionnel d'avec les autres types de justification pour le maintien de l'excision.

"Le chef de notre village a même juré que l'excision ne se pratique plus. Donc il ne le fait plus. S'il le faisait, nous l'aurions su» (focus jeunes hommes, Zoundwéogo)

Le contact avec l'extérieur et d'autres valeurs a permis dans certains cas un changement de comportement. Il permet de mieux confronter ses idées et affermir sa décision.

«Les gens voyagent, ils vont partout. Les jeunes qui voyagent ramènent des informations. Ils expliquent aux gens et les sensibilisent. C'est pour cela qu'ils ont abandonné définitivement »(Leader communautaire homme, Oudalan)

Les uns et les autres reconnaissent que le niveau d'instruction et la position sociale sont des facteurs majeurs de lutte et d'opposition à la pratique. Considéré comme un dépositaire d'informations, la personne instruite constitue une référence et met tout en œuvre pour éviter l'excision de la fille.

"Lorsque tu n'est pas instruit, tes yeux ne sont pas ouverts. Ceux qui ont les yeux ouverts ont été à l'origine pour qu'on puisse sensibiliser les autres petit à petit, jusqu'à ce qu'ils comprennent " (Leader homme, Houet)

Ainsi il existe un changement dans les valeurs et normes au contact avec l'extérieur, la modernité et l'instruction. Cela crée un environnement ou la non excision n'est plus une valeur. 


\section{Les avantages relatifs de la non excision}

La vue ou le vécu des séquelles constitue pour certains, une étape majeure dans la prise de conscience. Ces conséquences leur ont permis de voir les dangers réels encourus par les petites filles ce qui constitue un puissant détonateur de changement de comportement envers l'excision et de détermination à ne pas exciser leurs filles.

"Jusqu'à la fin de ma vie, je ne reculerai pas. Rien ne peut me ramener en arrière surtout que j'ai vécu la situation. J'ai vécu la situation et je ne peux pas oublier» (Institutrice, Kadiogo)

Les avantages économiques sont aussi retrouvés dans le discours des populations. Face aux conséquences sanitaires, les populations entrevoient de plus en plus les difficultés économiques engendrées dans la vie de couple au moment de l'accouchement. Si les adultes sont surtout regardant des effets immédiats, les jeunes par contre sont particulièrement sensibles à ces difficultés futures et ne souhaitent pas les subir dans l'avenir.

"Avec cette pauvreté, s'il faut encore payer beaucoup d'ordonnance et souvent faire les césariennes, ce n'est pas facile pour nous les hommes » (Focus jeunes mixtes, Zoundwéogo)

"La copine de mon ami a été opérée pour ces deux accouchements. Tout cela est dî au fait qu'elle était excisée. Si à chaque accouchement tu dois faire une opération qui coûte entre $75.000 \mathrm{~F}$ et $90.000 \mathrm{~F}$, si tu n'as pas cette somme tu seras obligé de ne plus avoir d'enfants. Mais si la fille n'est pas excisée, cela n'allait pas arriver» (focus adultes mixtes, Houét)

\section{La compatibilité avec les convictions religieuses}

L'appartenance à la religion chrétienne, notamment protestante constitue un facteur important d'abandon de l'excision. La quasi totalité des enquêtés de confession protestante est défavorable à l'excision, qu'ils soient leader ou simple pratiquants. Chez les leaders communautaires d'obédience catholiques par contre, on assiste parfois à un conflit entre leur fonction de gardien de la tradition et leur croyance religieuse chrétienne.

"Mes parents sont des protestants. On avait dit qu'on m'exciserait mais ma mère a refusé que je sois excisée » (récit de vie fille non excisée, primaire, célibataire, Gourma)

"Au début, on a commencé à parler de l'excision à l'église catholique. En tant qu'élève catéchiste, je revenais faire le compte rendu au chef du village »(catéchiste, Zoundwéogo)

L'essai et l'observabilité

Dans la perspective des avantages véhiculés actuellement, plusieurs personnes ont préféré adopter la non excision, avec néanmoins la crainte de conséquences éventuelles chez certaines personnes âgées.

"Les enfants qui restent ne sont pas excisées. Si elles n'ont rien comme problème, c'est vous qui aurez raison. Mais s'ils tombent malades et qu'ils meurent, nous allons appeler vos noms en pleurant " (Personne ressource, Homme, 83 ans, Gourma)

\section{Les indicateurs de changement de comportement}

Disparition des mécanismes de renforcement

Les poèmes et chants glorifiant la fille excisée ne semblent plus être utilisés. Seule les femmes plus âgées s'en souviennent encore. Parmi les jeunes générations, ce type de connaissance a disparu. 


\section{L'atteinte de la masse critique dans la communauté}

Si tous reconnaissent que la masse des personnes ayant abandonné la pratique est assez importante, ils divergent néanmoins quant à l'atteinte de la masse critique qui permettrait l'effet d'entrainement de groupe.

En terme de changement d'attitude, cette masse critique est largement atteinte. Ce changement d'attitude se reflète par des déclarations à haute voix de l'abandon de la pratique, partout ou l'enquête a été menée. Il y a une nette déclaration de ne plus vouloir exciser les petites filles. C'est surtout les jeunes qui soutiennent que cette masse critique a été dépassée. Ces jeunes constituent les parents de demain, ce qui permet de croire que le niveau va évoluer constamment vers un déni total de la pratique.

"Pour les autres villages je n'en sais rien, mais ici je peux dire que ceux qui ont abandonné la pratique sont plus nombreux. Ceux là qui ont abandonné sont plus nombreux que ceux qui n'ont pas abandonné » (Pasteur, Zoundwéogo)

«Ceux qui ont abandonné sont deux fois plus nombreux que ceux qui n'ont pas abandonné. » (Leader communautaire, Houet)

Les populations reconnaissent que l'inertie des phénomènes démographiques ne permet pas de mesurer actuellement le changement au sein des populations de filles, étant donné que celles qui sont non excisées ne sont pas encore assez nombreuses pour influencer les chiffres. Cependant avec les années le changement sera de plus en plus perceptible.

"Ce sont celles qui sont excisées qui sont plus nombreuses. L'abandon étant récent, les non excisées sont moins nombreuses »(Catéchiste, Zoundwéogo)

"Les filles non excisées ne peuvent pas être plus nombreuses, parce que vu les générations qui sont passées.

Leur groupe va en croissant parce que les gens abandonnent du jour au lendemain » (agent technique de

l'agriculture, Oudalan)

\section{Des données réelles au niveau des formations sanitaires}

Les entretiens avec les agents de santé ont permis de faire un constat. Ils reconnaissent que beaucoup de petites filles qui viennent pour des consultations ne sont pas excisées. Parmi les femmes qui viennent pour les accouchements, ils rencontrent également des femmes non excisées; un constat très remarqué dans la région du Nord.

«On a reçu des filles qui sont venues, elles ne sont pas excisées » (Agent de santé, Gourma)

\section{La mise en œuvre de la décision d'abandonner la pratique}

Comme le présente le modèle de diffusion de l'innovation de Rogers, la mise en œuvre de la décision d'abandonner l'excision répond à certains éléments : la visibilité de cette décision peut se manifester de plusieurs manières, le consensus doit être atteint a travers un certain processus. 


\section{L'affirmation de la décision de ne plus pratiquer l'excision}

Au Burkina, l'affirmation de cette décision est rarement publique. Elle est en général individuelle et consiste à assumer tout seul la non excision de ses propres filles. Les discussions sur l'excision sont occasionnelles et ont surtout lieu dans l'environnement immédiat, c'est-à-dire dans l'entourage familial. Les actions publiques sont surtout le fait des leaders communautaires ou religieux, dans le cadre de sensibilisations de masse.

«Pour l'entourage familial, j'en parle permanemment» (Femme 48 ans, catholique, non favorable à la pratique de l'excision Houet).

Cependant il faut reconnaitre qu'au courant de ces dernières années, et suite aux stratégies d'implication des leaders et des groupes socioprofessionnels, on a pu assister à des prises de positions publiques de leaders sur la nécessité d'arrêter la pratique de l'excision. Cela a été largement relayé par les média.

\section{L'atteinte du consensus}

Il n'existe pas de façon ouverte un consensus au niveau de la communauté. Le caractère individuel de la pratique rend le consensus sous-jacent, sans véritable expression, sauf dans l'Oudalan où il y a visiblement un changement social perceptible.

«C'est progressivement que les mentalités ont changé avec la sensibilisation. Nous ne nous sommes pas levés un jour pour nous réunir et Sinon, ce n'est pas dire que nous nous sommes réunis un jour pour mettre fin à l'excision. C'est petit à petit que les mentalités ont changé » (Focus adultes mixtes, Houét)

"Petit à petit c'est fini. Si tu sais que tu vas faire la chose pour qu'elle se retourne contre toi alors abandonne-là. Fais comme les autres pour qu'elle finisse. (Focus hommes adultes, Kadiogo

Dans certains cas, ils n'hésitent pas à mettre en application les menaces en dénonçant les contrevenants.

«Il y a une vieille qui voulait exciser plus de dix filles à Kombissiri. J'ai demandé à ma grand-mère et elle a dit qu'elle ne pouvait pas intervenir. Donc, j'ai appelé le SOS excision de façon anonyme et j'ai indiqué l'endroit. Et ils sont venus. (Focus élèves, Kadiogo)

\section{Les stratégies pour éviter l'excision des filles}

En plus des menaces de dénonciation, l'isolement des filles de milieux à risque semble être la stratégie la plus utilisée pour éviter leur excision. Un isolement qui va parfois jusqu'à la rupture de liens entre parents de la même famille.

"J'ai dit à ma maman que si j'entends encore qu'elles vont exciser une fille je vais les dénoncer. Mes enfants ne seront jamais excisées. " (Récit de vie fille excisée, Ouagadougou, Kadiogo)

"J'ai souvent dit à ma mère. Si tu l'emmènes chez l'exciseuse, aujourd'hui tu dors à la gendarmerie. Chez nous, on a réussi à ne pas faire exciser les filles »(Prêtre catholique, Kadiogo).

\section{La confirmation de la décision de ne plus exciser}

Cette confirmation est une étape cruciale dans le processus de changement social, puisque permettant le maintien dans la durée de la décision de ne plus exciser les filles.

\section{La discontinuité éventuelle et le retour à l'excision ?}

Vraisemblablement, la loi apparaît comme un puissant moyen de dissuasion. Dans la zone du Nord par contre, la position des responsables religieux reste prépondérante dans une éventuelle 
reprise de la pratique. Aussi, la baisse ou l'absence des activités de sensibilisation sont perçues comme signifiant l'abandon de la lutte et encourageraient à reprendre l'activité. Pour d'autres, le comportement incontrôlé des filles et/ou la recherche effrénée de filles excisées par les hommes (dans le cadre du mariage) les conduiraient à reprendre l'activité pour éviter le célibat définitif des filles.

"C'est à cause de la peur de la loi, la répression, la prison qu'ils ont abandonné. Si on abolit cette interdiction, il y a des gens qui vont recommencer à exciser leurs filles »(focus adultes mixtes, Houét)

\section{Les projections sur l'adoption de la non excision}

Pour beaucoup, notamment les jeunes, l'excision ne peut plus reprendre de l'ampleur, vu l'actuel niveau de déclin de la pratique et la prise de conscience des populations. Aussi, l'offre et la demande faiblissent sous la pression de 1

a loi et la disparition des personnes âgées. Pour la jeune génération, 'excision subit l'influence des goûts et modèles occidentaux nouveaux quant au processus de mise en couple tandis que la loi continue d'être un leitmotiv d'abandon, même sans conviction.

"Beaucoup de garçons ont pris des filles non excisées, parce qu'on excise plus les filles. Donc si tu fais la cours aux filles et il n'y a pas d'excisées, où vas-tu aller les chercher? Les non excisées deviendront les valorisées comme les excisées l'étaient dans le passé. » (jeunes mixtes, Kadiogo)

\section{L'adoption tardive de certains résistants}

Sur la base de cas réels, notamment l'arrivée de plus en plus de filles non excisées à l'âge de la maternité et qui accouchent sans difficultés majeures, certains sceptiques ont pu changer de comportement. Pour les jeunes, le fait que cela ne remette pas en cause la fécondité et la reproduction de la famille a permis et permettra encore à des résistants au changement d'adopter la non-excision comme innovation majeure.

"Présentement, je ne vois même pas d'avantages parce que je vois des filles non excisées présentement et qui arrivent à mettre au monde des enfants. Or pourtant, nos ancêtres disaient qu'une femme non excisée ne pouvait pas accoucher. Même ma fille, on avait pas le choix, la première a été excisée. Mais les deux restes, jamais. Sa petite sœur a un enfant comme elle, et sans problème (chef coutumier, 58 ans, niveau primaire, Kadiogo).

\section{Les contraintes à l'adoption de la non pratique de l'excision}

Les contraintes restent néanmoins encore vives dans certaines communautés.

\section{La persistance des résistances}

Le niveau de conviction conduit les adeptes de l'excision à prendre des risques, malgré la loi. Au-delà du risque encouru, l'utilisation de subterfuges, le grand secret et la discrétion ainsi que la complicité sont forcément nécessaires pour le succès de telles initiatives. La cohésion du groupe est probablement un facteur permettant la perpétuation et la solidité des convictions. Certaines zones (un des villages enquêté du Gourma) ou certains groupes ethniques (les Ouoba) semblent avoir plus de résistants que d'autres. Cependant, parmi toutes les zones enquêtées, ces résistances exprimées ouvertement sont devenues très marginales.

A - Les raisons sociales et psycho-sexuelles

Les raisons sociales et psycho-sexuelles sont les raisons les plus avancées par toutes les communautés, dans toutes les provinces, dans toutes les localités. 
Le besoin d'acceptation sociale. Pour certains leaders, il est difficile d'allier le suivi de la tradition et l'abandon de l'excision, vu que la tradition suppose l'excision des filles, ce qui crée parfois un sentiment de manque de repère solide. Dans les faits, on assiste chez certains leaders à un silence presque complice en cas de pratique de l'excision. En effet, la dénonciation par exemple expose son auteur à des sanctions sociales telles que l'exclusion sociale, ce qui instaure un climat de peur des réprimandes ou des représailles des adultes.

"La marginalisation rend malheureux. C'est pour cela que tout le monde pratique, même s'il faut entrer sous le sol »(Femme, 45 ans, Favorable à la pratique de l'excision, Houet)

Le désir de contrôle de la sexualité des femmes. C'est ici l'élément le plus important et le plus fédérateur des différentes populations. Ce facteur psycho-sexuel est celui qui induit le comportement en matière d'excision, et conduit à la stigmatisation et à la discrimination des filles non excisées. Il permet parfois le rapprochement entre les jeunes et les adultes et renforce le caractère sacré du mariage et lui donne toute son importance, dans un milieu très souvent tiraillé entre le modernisme et la tradition.

"Nos parents nous ont toujours dit que lorsque tu mets au monde une fille et que tu ne l'excise pas, elle aimera les hommes. Elle devient insatiable, elle court derrière les garçons, Quand elle a envie de faire l'amour, elle ne peut pas se maîtriser »(Focus adultes mixtes, Houét).

Au delà du mariage et des avantages qui y sont liés, la recherche de la chasteté et de la virginité reste encore une préoccupation des parents. A terme, ceux-ci restent convaincus que l'excision est le seul remède qui peut garantir ce type de comportement. C'est pourquoi, les citadins proposent des solutions alternatives qui supprimeraient uniquement les conséquences sanitaires. Une chasteté qui pourrait sauver l'honneur familial et garantirait le succès du mariage et éloignerait le spectre de la répudiation, la plus grande des hontes pour la fille et sa famille.

"Même ta propre mère n'est pas respectée. Chaque fois qu'il y a un groupe de femmes au puits, on la critique. Donc pour une fille qui n'est pas excisée, sa famille est marginalisée. Parfois, on estime que cette famille a une mauvaise éducation »(Institutrice, Kadiogo).

La persistance des croyances. Certaines croyances s'expriment parfois de manière forte et continuent de convaincre certains de la nécessité de poursuivre la pratique. Le clitoris par exemple serait un organe qui ne cesse de grandir et masculinise la femme. Il est également considéré comme un objet vampirique qui boit le sang de l'enfant à la naissance. Il serait la source de maladies, de sorte à créer un état de morbidité permanente chez la femme. On notera que ces croyances relèvent parfois de leaders d'opinion influents et paradoxalement de personnes instruites. Le milieu urbain aussi se fait malheureusement le relais de ces croyances.

"Je dis ceci : si tu marche avec cette affaire qui pend entre tes cuisses, aucun homme ne viendra vers toi.

Personne ne veut d'un hermaphrodite" (leader religieux, Ouagadougou)

"Une femme enceinte peut perdre son enfant car le clitoris peut absorber le sang de l'enfant et provoquer sa mort ». (Focus Femmes Adultes Tanghin, Kadiogo)

Néanmoins, avec la sensibilisation, les populations acquièrent de nouvelles connaissances qui sont mises à profit pour développer des arguments contraires. Ces nouveaux arguments touchent aussi bien les références à la coutume, aux maladies et aux vers. Dans le Gourma par exemple, l'argumentaire contraire est très bien développé.

"Le problème c'est qu'une fille non excisée est fréquemment malade, et il faut l'excision» (Leader communautaire, Zoundwéogo) 
"Si tu laisses ton enfant nu, que ce soit en saison sèche ou pluvieuse, si elle est assise à terre, il y a beaucoup

de germes qui sont à terre » (leaders religieux, catholique, Gourma)

La nécessité religieuse. La référence à la religion émane surtout des populations musulmanes. La majeure partie des leaders religieux musulmans, les leaders communautaires musulmans et une partie des personnes ressources de religion musulmane qui ont été rencontrés sont favorables à la pratique de l'excision. Dans certaines langues comme le Dioula, les termes de désignation de la pratique de l'excision "Ka Selidjita, Ka do selidji là" font référence à l'initiation à la prière.

"Un musulman ne va pas accepter d'épouser une femme non excisée. C'est difficile 》 (Leader communautaire musulman, 63 ans, Kadiogo)

Néanmoins, les arguments religieux sont de plus en plus rejetés, même dans les zones fortement islamisées telles que l'Oudalan où les femmes semblent particulièrement avoir été marquées par le message de certains chefs religieux.

\section{$B$ - Les mécanismes de renforcement communautaire}

Un certain nombre de mécanismes existent pour amener les familles à exciser leurs filles, dans un but de cohésion sociale. Au Burkina, trois mécanismes importants sont à noter : la peur de représailles surnaturelles, le divorce et/ou le refus de marier des filles non excisées et l'obligation d'exciser des femmes de communautés n'excisant pas.

«Il y a beaucoup de vieilles femmes qui ont beaucoup de médicaments. Si tu te mêles de leurs affaires, elles peuvent te tuer aussi. Ce sont les fétiches, ça ne se comprend pas » (Focus jeunes mixtes, Houet)

Mais ces mécanismes utilisent une nouvelle forme d'expression. Par exemple, il n'apparaît pas d'opposition ouverte au mariage des filles non excisées, mais plutôt une forme beaucoup plus subtile pour décourager ceux qui voudraient s'y engager. La ville (avec son style de vie sans limites) apparaît comme le lieu où cette forme de pression se fait le plus sentir.

Cependant, dans certaines zones comme l'Oudalan, on assiste plutôt à des mariages interethniques sans trop grande difficulté.

«Nous cohabitons avec eux, nous adoptons leurs coutumes, nous nous marions avec eux et ils se marient avec nous. Nous avons trouvé que ce n'était pas une chose obligatoire " (focus adultes femmes, Oudalan)

\section{La justification de la poursuite de la pratique}

\section{Les avantages relatifs de l'excision}

Les adultes de façon générale, ont du mal à comparer les avantages relatifs de la non excision, étant entendu que leur âge ne permet pas toujours d'expérimenter l'inconnu et ou de mesurer le degré et l'étendue des avantages actuellement diffusés. Les jeunes femmes, notamment en milieu urbain, s'interrogent surtout sur les avantages relatifs sur le plan sexuel. Face aux arguments des séquelles sanitaires de l'excision, les partisans de l'excision arguent que l'excision ne peut être mise en cause, vu que les opérations chirurgicales ne sont pas non plus sans danger pour les patients. Pour eux, il faudrait entre autres incriminer l'inexpérience de la praticienne.

"C'est peut être celle qui n'est pas excisée qui est mieux. Nous entendons les jeunes le dire, mais nous n'en savons rien, parce que nous sommes avec des excisées »(Leader communautaire, 56 ans, Oudalan) 
La compatibilité avec les valeurs traditionnelles

Face aux campagnes massives, à la volonté politique et à l'application de la loi, les partisans de l'excision crient à l'injustice, au danger du mimétisme culturelle, notamment celle des valeurs occidentales qui menacent les traditions et les valeurs traditionnelles. C'est pourquoi ils adoptent une position défensive qui se traduit dans les faits par un double langage, la recherche d'argumentaires aussi bien dans le domaine moderne que traditionnel et/ou la sensibilisation à contre courant.

«Ils tenteront tout mais ne réussiront pas à faire disparaître la tradition » (Responsable administratif villageois, Gourma)

\section{L'observabilité}

Pour ne pas être de cas déviants, l'attitude des adultes consiste parfois à observer et attendre les résultats de ce changement, c'est-à-dire ce qui adviendrait des filles et femmes non excisées, notamment si leur reproduction et partant celle du groupe ne sera pas mis en danger. Cette vision du futur semble hanter essentiellement les anciens dans le Gourma, parce que impossible à prévoir. Une observabilité qui semble s'installer dans la durée afin de suivre toutes les phases de l'évolution du groupe social.

"Je n'ai pas encore vu une fille non excisée qui s'est mariée Si deux à trois se marient et que tout va bien pour elles, là nous allons suivre. S'il font et qu'on voit que notre famille peut exister sans excision, nous remercierons Dieu »(Responsable administratif villageois, Gourma,)

\section{La complexité de la non excision}

Le sentiment du devoir à accomplir : Face aux conceptions liées aux aspects psycho-sexuels, exciser sa fille apparaît comme un devoir des parents et s'y plier donne le sentiment d'avoir accompli sa tâche de protection, de garantie de santé, etc. A leurs yeux, cet acte mériterait plutôt la reconnaissance des filles et femmes excisées.

"Les filles ne savent pas que tout ce que font leur mère c'est pour elles " (Focus hommes adulte,

Kadiogo).

L'ambiguïté du rôle des relais communautaires : ces relais ne bénéficient pas toujours d'un environnement favorable à la sensibilisation. Certains font face à des alliances et liens sociaux difficiles à stabiliser en cas d'accusation, d'indexation, de conflit social. Aussi, le choix des relais s'avère être une opération stratégique pour les communautés, afin de s'assurer le contrôle de leur actions pour bénéficier de leur bienveillance en cas d'excision d'un des membres.

Le mauvais exemple des intellectuels et des citadins : ils se réfèrent très souvent au village pour solliciter l'excision de leur progéniture et contribuent ainsi à semer le doute dans l'esprit de ceux qui ont abandonné ou qui s'apprêtent à le faire.

«Le comble c'est qu'il y a des enfants qu'on a fait venir de Ouagadougou pour l'excision ici » (agent de police, Bazèga)

La loi de l'offre et de la demande : le métier d'exciseuse étant en disparition, celles qui sont reconnues comme tel sont très demandées. Celles de la capitale Ouaga semblent être les plus sollicitées, probablement à cause de la répression qui y est très forte et des moyens financiers dont disposent ces citadins pour s'assurer le service.

«Moi aussi je pouvais me cacher pour exciser. Les gens viennent me solliciter mais je refuse» (ex-exciseuse, 62 ans, Ouagadougou) 
Les liens entre non excision et libertinage sexuel actuel : un lien très étroit et direct est établi par l'ensemble des populations des différentes zones entre la liberté de mouvement, le libertinage sexuel des filles et la non excision. Elles se refusent à réduire cette situation au problème d'éducation (de manière générale), mais incriminent le développement des médias, notamment la télé et ses images parfois obscènes. Une idée particulièrement partagée chez les citadins. Un environnement qui renforce et conforte l'image négative véhiculée des filles et femmes non excisées. Cependant, les jeunes se démarquent de plus en plus de cette vision négative de la fille non excisée.

«Avant, nos pères pouvaient voyager pendant 2 ou 3 ans en Côte d'Ivoire par exemple. A leur retour, ils trouvent leur femme qui les attend. Mais aujourd'hui, si tu sors sans ta femme, 3 mois c'est déjà beaucoup. Elle va te tromper» (focus jeunes mixte, Kadiogo).

"Une fille non excisé peut coucher avec 10 hommes la même nuit. Si une fille vient chez toi de nos jours et que tu ne couches pas ave elle, elle ne revient plus. C'est cela qui amène les grossesses non désirées » (Focus adultes hommes, Kadiogo)

"Actuellement, nous avons honte de nos enfants. Les filles et les garçons se serrent dans les bras coincés sur les murs »(Focus adultes mixtes, Houét).

\section{La mise en œuvre de la volonté de continuer l'excision des filles}

Renforcés par ces différents arguments, les résistants mettent en place plusieurs stratégies pour poursuivre l'excision.

L'âge à l'excision: Les petites filles sont maintenant préférées aux autres parce qu'elles sont plus dociles, plus faciles à camoufler, de faibles résistance et susceptibles d'oublier la douleur et les traumatismes éventuels. Cette tendance est fortement présente dans le Houet où la population est en majorité musulmane

La période : Aujourd'hui, le choix se porte sur la période hivernale où les familles s'attèlent dans les champs, ce qui ne favorise pas beaucoup les occasions de rencontre et de discussion. Elle serait propice grâce aux tiges de mil et de maïs qui permettent de se camoufler. Au Bazèga, on a pu noter que la nuit est un allié efficace pour éviter les regards indiscrets.

Les complicités : Dans le Houet particulièrement, les cas et les stratégies mises en œuvre sont nombreuses : excision et soins à domicile, déplacement vers les villages reculés, etc. La complicité ambiante dans certains milieux fait que la dénonciation n'est pas toujours évidente et systématique, encore moins lorsque les uns et les autres se connaissent, décident ensemble et se soutiennent mutuellement. 


\section{Discussion ET CONCLUSION}

Le niveau général de la pratique de l'excision au Burkina reste quelque peu élevé dans la majeure partie des provinces du pays, avec des différences ethniques parfois prononcées. Le niveau d'instruction inhibe la volonté d'exciser les filles tandis que l'appartenance à la religion musulmane augmente le risque pour la jeune fille de se faire exciser.

Cependant, l'examen de la pratique dans les cohortes indique un écart important entre mères et filles aux mêmes âges, avec un niveau beaucoup plus faible chez les petites filles. De plus, les attitudes sont de plus favorables à l'abandon de la pratique. Les données secondaires indiquent par contre que 23\% des filles peulh (dans le Nord) de 0-4 ans sont excisées tandis que les données qualitatives qui ont été recueillies sur le terrain indiquent plutôt une disparition de la pratique dans l'Oudalan. Cette contradiction n'est qu'apparente si on considère que dans l'Oudalan, les populations Bella sont reconnues comme n'excisant pas leurs filles. Aussi, les uns et les autres s'accordent à dire que l'abandon de la pratique est assez récente, de même que les informations récoltées ont été corroborées par l'observation de quelques petites filles de moins de 10 ans.

Parmi les principaux facteurs de changement de comportement, la loi apparait comme la plus dissuasive. Cependant, le contexte national est tributaire des activités de sensibilisation de masse, de même que les multiples interventions sur le terrain qui accompagnent cet engagement politique et judiciaire. Ces interventions, soutenues par les partenaires au développement et mises en œuvre par les structures et organisations locales, ont fortement influencé les attitudes et le niveau de connaissance des populations. Concomitamment, en combinaison ou en symbiose avec la loi et les interventions, les facteurs endogènes tels que le niveau d'instruction, notamment des filles (accès aux écoles et les possibilités de poursuite des études), le contact interne ou externe avec d'autres peuples ainsi que la progression des migrations, de l'accès aux mass média, surtout la radio et la télé contribuent, sinon déterminent le changement social dans le sens de l'adoption de la non pratique de l'excision comme comportement nouveau. Des changements qui s'inscrivent désormais dans le système social actuel. C'est pourquoi, il est important de ne pas abandonner les activités de sensibilisation ou amoindrir l'application de la loi, qui serait dans ces conditions considérées comme un signal fort pour un retour à la pratique. Ce processus de changement social est fortement soutenu par les jeunes qui non seulement se démarquent de plus en plus des aînés, mais qui adhèrent aux nouvelles valeurs, notamment occidentales, véhiculées en grande partie par les mass média.

En résumé, il est à noter que le rôle de l'excision dans la fécondité de la femme et sa reproduction, éléments cruciaux pour les populations africaines fortement pro natalistes (à l'exemple du Burkina) émerge fortement. C'est pourquoi, s'attaquer aux croyances sur l'excision passe donc par les rassurer sur le fait que la non-excision des filles ne mettra pas en danger leur capacité reproductive et ne mettra pas en danger la survie démographique de la communauté.

Force est de reconnaître néanmoins que des réorientations de stratégies sont nécessaires au regard des différences géographiques du niveau de la pratique de l'excision. Si dans certaines zones cohabitent des groupes ethniques parmi lesquels il y'en a qui n'excisent pas, dans d'autres par contre, ce sont des sous groupes ou familles qui ne le font pas ou ont depuis longtemps 
abandonné la pratique. En témoigne les femmes qui n'ont pas été excisées avant la loi et qui accouchent aujourd'hui et qui ne sont pas excisées. La loi pourrait bien sûr y avoir contribué mais cela pourrait aussi traduire une détermination des familles, des couples ou des personnes a ne pas exciser bien avant l'adoption des mesures légales d'interdiction. Les raisons pourraient être liées aux activités de sensibilisation ayant pris effet avant la loi de 1996 ou liées à des convictions personnelles découlant de facteurs endogènes et/ou exogènes.

Des réorientations des stratégies sont nécessaires également face à la persistance des croyances erronées et de la persistance de la pratique. Malgré les signes tangibles de la baisse continuelle de la pratique avec l'arrivée de la cohorte de filles non excisées sur le marché matrimonial et les accouchements, les stratégies développées par les opposants au changement sont diverses et contribuent bien que faiblement à une sous-estimation du niveau actuel de la pratique. Ce d'autant plus que la majeure partie des populations est très bien informé de l'interdiction de l'excision et de l'incarcération prévue en cas de délit constaté.

En dehors de la religion musulmane qui demeure toujours un facteur de la poursuite de la pratique, les raisons principales évoquées qui fédèrent toutes les couches de la population sont celles relatives aux aspects psycho-sexuelles. Si pour les résistants elles sont des raisons de la poursuite de l'excision, pour ceux qui ont adopté la non excision, elles s'expriment comme des craintes pour la santé de la reproduction et la perpétuation démographique du groupe social. Ainsi, il s'établit un lien entre débauche sexuelle et excision, ce d'autant plus que le modernisme et la globalisation de la culture poussent les jeunes, surtout les filles à certains comportement (sexualité, vestimentaire, ...) systématiquement attribués à la non excision. C'est dans ces conditions que la stigmatisation des filles non excisées est grandissante, instaurant de ce fait un doute dans l'esprit de ceux ayant laissé leurs filles non excisées. Des raisons principales qui ne sont pas très bien explicites, mal abordées ou absentes dans les différents outils et messages développés, notamment en ce qui concerne la responsabilité des parents dans leur rôle d'éducation des filles. Pour y faire face, les canaux de diffusion des mécanismes de renforcement communautaire ont changé de forme d'expression pour être plus subtiles et parfois très efficaces.

Le caractère clandestin, familial et individuel actuel de l'excision entraine une faiblesse de l'affirmation publique de la décision d'abandon. Une situation qui ne permet pas au modèle de changement social développé de mieux appréhender le processus d'adoption communautaire (ou sur le plan national) de l'innovation dans le contexte du Burkina. Il ne prend pas non plus en compte les divergences culturelles mais peut être adapté à un milieu spécifique homogène, notamment dans le cadre d'une intervention ciblée. 


\section{Recommandations}

Quelques recommandations sont faites dans le but d'améliorer les stratégies pour les rendre plus efficaces pour une promotion de l'abandon de l'excision. Il faudra :

1. Evaluer et réviser les messages en les adaptant aux aspects psycho-sexuels, des messages venant en support des parents ayant abandonné l'excision, en support aux filles non excisées et en direction des filles excisées, des messages adaptés au contexte actuel et au public cible ;

2. Utiliser davantage du matériel IEC et des canaux permettant de diffuser des messages sous forme visuelle mettant en avant les souffrances des personnes excisées ;

3. Rassurer les communautés sur le fait que la non excision des filles ne mettra pas en danger leur capacité reproductive et ne mettra pas en danger la survie démographique de la communauté ;

4. Encourager et engager des discussions sur la santé sexuelle et reproductive des filles, notamment en rapport avec l'éducation parentale et la communication intergénérationnelle ;

5. Mettre en place une stratégie de communication intergénérationnelle sur l'excision et la santé;

6. Cibler plus les jeunes dans les stratégies et les impliquer en les utilisant comme agent de changement de comportement ;

7. Cibler les enfants et les jeunes à travers l'extension de l'enseignement des modules sur l'excision dans le système éducatif (primaire, secondaire et supérieur) pour préparer leur avenir et déjouer les stratégies de contournement de la loi ;

8. Renforcer les interventions dans certaines zones : dans le Gourma, il s'agira de mieux les convaincre sur la sécurité démographique tandis que dans le Houet, il faudra renforcer les stratégies de dénonciation ;

9. Renforcer la sensibilisation de proximité des relais communautaires en leur apportant l'appui nécessaire en termes de capacités et de valorisation du travail ;

10. Étendre et intensifier les patrouilles de sensibilisation pour une plus grande efficacité, notamment pendant la période des vacances et la période hivernale ;

11. Renforcer la sensibilisation des groupes socioprofessionnels (magistrats, gendarmes, policier, avocats,...) pour une application rigoureuse de la loi sur la pratique de l'excision ;

12. Intensifier le plaidoyer auprès des partenaires pour un renforcement des capacités du CNLPE aussi bien au niveau central que déconcentré ; 
13. Utiliser les média, particulièrement les radios communautaires pour identifier les déviants positifs (surtout les adultes urbains et intellectuels) et les encourager à faire des témoignages à visage découvert ;

14. Encourager les femmes ayant eu des effets négatifs de l'excision à faire des témoignages, particulièrement celles qui viennent pour les réparations des séquelles ;

15. Renforcer le système de suivi-évaluation des interventions sur le terrain pour plus d'efficacité ;

16. Impliquer les élus locaux dans la promotion de l'abandon de la pratique de l'excision ;

17. Organiser des rencontres de concertation conjointes entre comités des pays frontaliers en vue de venir à bout de la pratique transfrontalière ;

18. Encourager les dignitaires religieux à se positionner publiquement et clairement sur la question de l'excision ;

19. Vulgariser le contenu des articles 380 à 382 du code pénal réprimant l'excision ; 


\section{REFERENCE BIBLIOGRAPHIQUE}

Bationo E. 1991. Les mutilations sexuelles au Burkina Faso : Description et stratégies de lutte, le cas de l'excision. Mémoire ENAM, Ouagadougou, 102 p

Centre pour le droit et les politiques en matière de santé et de reproduction (CRLP). 2000. Les droits de la personne: un outil pour combattre les mutilations génitales féminines, Guide pratique au service des militants avril, 60 pages

CIAF. 2003. Tolérance Zéro à la MGF, Agenda commun pour l'action en vue de l'élimination des Mutilations Génitales Féminines 2003-2010, février, 30 pages

Comité Nationale de Lutte contre la Pratique de l'Excision (CNLPE). (1999). Plan d'action de lutte contre la pratique de l'excision (1999-2003),Ouaga, 39 pages + Annexes.

Comité Nationale de Lutte contre la Pratique de l'Excision (CNLPE) 2003. Bilan des actions du CNLPE 1990-2003, mai, 23 pages

Comité Nationale de Lutte contre la Pratique de l'Excision (CNLPE). 2004. Rapport narratif des patrouilles de sensibilisation et de dissuasion réalisées par vingt deux (22) brigades de gendarmerie, Octobre, 10 pages

Comité Nationale de Lutte contre la Pratique de l'Excision (CNLPE). 2005 : Rapport de la rencontre de concertation des acteurs de la mise en œuvre des activités financées par l'Ambassade Royale des Pays -Bas

Congo Zakari et al. 2004. Expérience d'un Programme d'Education de Base au Burkina Faso, Population council, Mwangaza, UERD,TOSTAN, GTZ, Ouagadougou, 51 pages

Conombo Joseph Issoufou (1989) : M'ba Tinga, Traditions des mossé dans l'Empire du Moogho-Naba, L'Harmattan, Paris, 185 pages.

Creel Liz (2002) : Abandonner l'excision, Population Reference Bureau, 33 pages.

Dera Lassané, Ilboudo François, Kaboré Idrissa, Ouattara Adama, Yé Aminata (1997) : Enquête nationale sur l'Excision au Burkina Faso, INSD, Ouaga, 62 pages + annexes.

Gnanou Octavie (2001) : Les fondements socio-culturels de la prévalence de l'excision au Burkina Faso : Etude comparative de quatre groupes ethniques, mémoire de licence, UCL, 80 pages

Handnaba L. 1988. La pratique de l'excision et les mentalités modernes chez les mossi à Pabré, mémoire Diplôme Assistant Social, Cotonou : 111p

Isett Susan et Toubia Nahid. 1999. Learning About Social Change, A research and Evaluation Guidebook Using Female Circumcision as a Case Study, Rainbo, New York, 109 pages 
Jackson F. Elisabeth, Akweongo Patricia, Sakeah Evelyn, Hodgson Abraham, Asuru Rofina, Phillips F. James. 2003. Women's Denial of Having Experienced Female Genital Cutting in Northern Ghana : Explanatory Factors and Consequences for Analys of Survey Data, "Policy Research Division Working Paper $N^{\circ} 178$. New York: Population Council

Kaboré Gisèle, Sobela Suzanne, Saloucou Lydia. 2002. Revue des politiques et des programmes sur la santé de la reproduction des adolescents au Burkina faso, UNFPA-Population Council, Ouagadougou, 98 pages.

Kaboré Yimian. 2002. Etude de base sur la pratique de l'excision dans 16 provinces du Burkina Faso, CNLPE-Coopération Pays-Bas, 77 pages

Kéré L., Tapsoba I. 1994. L'excision au Burkina, pratique et conséquences sur la santé, Ouagadougou : journal Eureka, $11 ; 16-33$ p

Kessler Bodiang, Claudia. 2003. La lutte contre les mutilations génitales féminines, défis et perspectives pour les programmes de santé ; première partie : une sélection d'approches, GTZ, février, 42 pages.

Konaté Alima. 1993. Les mutilations sexuelles féminines au Burkina Faso : le cas de la pratique de l'excision dans la société BWA de Houndé, Mémoire Sociologie, Ouagadougou, 61p

Konaté Alimata, 1998. Rapport de l'étude qualitative sur les mutilations génitales féminines dans douze (12) villages Plan du Sud-Ouest, Ouagadougou, Mai, non paginé

Konaté M. 1991. Les rites d'initiation chez les Kurumba du Luhrum, Mémoire Sociologie, Ouagadougou, $185 \mathrm{p}$

Laboratoire de Santé Communautaire. 1998. Etude participative pour l'identification des stratégies communautaires de lutte contre la pratique de l'excision dans le Bazèga, Série documentaire $n^{\circ} 22$, Ouagadougou, Décembre, 30 pages

Lamizana Mariam (1997): The campaign against Excision : Burkina Faso's experience, presentation in New York, September 18

Ministère de l'économie et du développement. 1993 : Enquête Démographique et de Santé du Burkina Faso,

ORC Macro, Calverton, USA, 296 pages

Ministère de l'économie et du développement. 1999 : Enquête Démographique et de Santé du Burkina Faso,

ORC Macro, Calverton, USA, 327 pages

Ministère de l'économie et du développement. 2003 : Enquête Démographique et de Santé du Burkina Faso, ORC Macro, Calverton, USA, 455 pages 
NEBIE Paul Stanislas (1999) : Collecte de données pour un processus de planification stratégique, Population Council, Rapport de recherche, 27 pages.

Nitiéma P. 1993. Les mutilations génitales féminines dans la ville de Ouagadougou: épidémiologie, évolution. Thèse Médecine nº 8 , Ouagadougou : 68 p

OMS. 1982. Circoncision féminine, Position et activités de l’OMS, Genève, 23 pages

OMS. 1986. Une coutume préjudiciable à la santé : l'excision féminine, Chronique OMS, vol 1, $\mathrm{n}^{\circ} 40: 33-38 \mathrm{pp}$

OMS. 1993. Mutilations sexuelles pratiquées sur les femmes : l'Assemblée Mondiale de la Santé en appelle à l'élimination de ces pratiques traditionnelles dangereuses, 1-3

OMS. 1996. Mutilations sexuelles féminines: Rapport d'un groupe de travail technique de l'OMS, Genève 17-19 juillet 1995, Genève, 30 pages

OMS. 1997. Faits et chiffres Mutilations Sexuelles Féminines, Presse OMS, n² : 1-2

OMS. 1997. Plan d'action régional pour accélérer l'élimination des mutilations sexuelles féminines en Afrique, Brazzaville, $59 \mathrm{p}$

Ouédraogo Amélie, 1998. Etude qualitative sur la pratique des mutilations génitales féminines dans dix (10) villages Plan de chacune des provinces du Bam et du Sanmatenga, Ouagadougou, juin, 48 pages + annexes

Ouédraogo Diénéba et al. 2006. Etude de base sur les mutilations génitales féminines (MGF) dans la région du Sud-Ouest du Burkina Faso, GTZ, Ouaga, Rapport d'enquête, 65 pages

Ouédraogo Diénéba, Kaboré Yimian, Sanon Edème. 2001. Etude de Base sur la pratique de l'excision dans cinq provinces du Burkina, GTZ, Ouagadougo, 84 pages.

Ouédraogo Idrissa et al. 2001. Etude sur la prévalence de l'excision au Burkina Faso, CNLPEOMS, 67 pages.

Ouédraogo Marie L. et Tapsoba Lydia. 2005. Evaluation interne du projet Lutte contre la Mutilation Génitale Féminine au Burkina Faso, Médicus Mundi Andalucia, Février, 75 pages.

Ouédraogo Saïdou, 2004 : Etude sur les droits des enfants et de la femme au Burkina Faso, Rapport d'étude, Ouagadougou, Novembre, Non publié, 30 pages

Population Council (2002): La recherche opérationnelle au service des programmes de campagne d'éradication de l'Excision, Rapport de réunion, Frontières en santé de la reproduction, Avril, Dakar, 40 pages 
Province du Yatenga et ONG Sentinelles. 1989. Séminaire national sur les pratiques traditionnelles affectant la santé de la femme: le cas de l'excision, rapport du séminaire, Ouahigouya 16-18 février, 86 pages.

Rahman Anika et Toubia Nahid. 2000 : Female Genital Mutilation, A guide to laws and policies worldwide, RAINBO, CRLP, 249 pages

Rogers Everett. 1995 : Diffusion of Innovations. Fourth edition, New York, NY : The Free Press, 127 pages

Sangli Gabriel et Ouédraogo Moctar. 2001. Enquête de prévalence sur la pratique de l'excision et l'impact des actions menées dans 7 provinces du Burkina Faso, CNLPE-UNICEF, 77 pages

Sawadogo Haoua. 2002. Les violences domestiques faites aux petites filles dans la ville de Ouagadougou, Burkina Faso, juillet, mémoire de fin de cycle, collège coopératif-AlpesMéditerranée, 108 pages + annexes

Sebgo Pascaline. 1999. Evaluation des initiatives contre les mutilations génitales féminines et proposition de la composante du projet supra régional au Burkina Faso, Rapport de mission, Ouagadougou, 108 pages

Secrétariat d'état à l'action sociale. 1988. Séminaire national sur les pratiques traditionnelles affectant la santé de la femme : le cas de l'excision, rapport du séminaire, Ouagadougou 26-28 mai, 78 pages.

Tarbagdo C. 1996. Lutte contre la pratique de l'excision au Burkina Faso : la contribution de la radio rurale, Mémoire CIERRO, Ouagadougou : 128p

Tarrab G., Coene ch. 1989. Femmes et pouvoirs au Burkina Faso, Québec, Paris, G. Vermette, Harmattan : 125p

Toé S. et Tamboura. 2001. Etude sur les connaissances, attitudes et perception des jeunes de 15 à 24 ans sur la pratique de l'excision: cas des departments de Banfora et de Manga, Cours International de Formation en Recherche-Action (CIFRA), Ouagadougou, 44 pages + annexes

Toubia Nahid, Rahman Anika (2000) : Female Genital Mutilation: A Guide to Laws and Policies Worlwide, New York, RAINBO, 249 pages.

Tougry L. P. Ester. 1998. Excision - tradition et modernité - causes et conséquences de la persistance de la pratique de l'excision en milieu moaga : cas de Wavugue et de Sabtinga, département de Pabré, mémoire de fin d'étude, Université de Ouaga (Sociologie), 76 pages + annexes

Traoré B., Valea C. 1993. Mutilations sexuelles, Regard n³0 : 8-12 
UERD (Unité d'Enseignement et de Recherche en Démographie), Direction, de la Santé de la Famille et Population Council. 1997. Rapport provisoire de l'enquête quantitative de Base, Ouagadougou, Burkina Faso, 78 pages

UERD (Unité d'Enseignement et de Recherche en Démographie). 1998. Community Health Laboratory, Evaluation Report, 69 pages

UNICEF. 1999. La lutte contre l'excision au Burkina Faso, Ouagadougou, février 41 pages

UNICEF. 2003. Activités du plan intégré de communication sur les mutilations génitales féminines et le code des personnes et de la famille, rapport synthèse, Ouagadougou, juin, 9 pages

World Health Organization (1999) : Female Genital Mutilation, Programs to Date : What Works and What Doesn't, A Review, 162 pages

Yoder P. Stanley, Noureddine Abderrahim and Arlinda Zhuzhuni. 2004. Female Genital Cutting in the Demographic and Health Surveys: A critical and Comparative Analys, ORC Macro, Calverton, 65 pages

\section{$\underline{\text { Les rapports }}$}

Batabe C. Mathias et Ahizinta R. Frédéric. 2002. Rapport des ateliers de planification des projets par objectifs des membres et partenaires du comité national de lutte contre la pratique de l'excision, Ouaga 18-22 mars et Bobo 2-6 avril, rapport des ateliers, tome I : Synthèse des travaux de planification des projets par Objectifs, différentes paginations

Belem Justine et Nougtara Gnoné. 2001. Rapport de formation en compétence Pédagogiques des formateurs en IEC/Excision, Kamboinsin 15-26 janvier, non paginé.

Enda-Acas. 1996. Séminaire sous-régional sur l'excision : échanges sous-régionaux et stratégies combinées, Ziguinchor du 29-31 janvier 1996, 135 pages.

Ministère de 1'Action Sociale et de la Famille. 1995. Rapport de la Session de formation en IEC/Excision, Koudougou 6-11 novembre, 22 pages, rapport général

Ministère de l'Action Sociale et de la Solidarité Nationale. 2004. Rapport de la formation des agents sociaux de neuf (09) provinces du Nord et de l'Est en IEC/Excision par l'ONG Medicus Mundi Andalucia, Ouaga 28 juin au 02 juillet.

Nibakure Isabelle. 1996. Rapport de l'Atelier d'auto-évaluation du comité national de lutte contre la pratique de 1'excision et des comités provinciaux, Bobo-Dioulasso 23-25 février, 28 pages + annexes.

Rapport du séminaire provincial de sensibilisation contre la pratique de l'excision, tenu à POprovince du Nahouri les 23-25 Août 1990, 46 pages. 
Sacko Bani (2003): Atelier de bilan et discussion stratégiques de la deuxième phase du projet MGF/GTZ au Burkina Faso, Coopération technique allemande, Bureau GTZ Ouagadougou, Rapport de synthèse, 20-21 novembre, 17 pages. 


\section{ANNEXES}

\section{Annexe 1 : Le schéma d'analyse du projet}

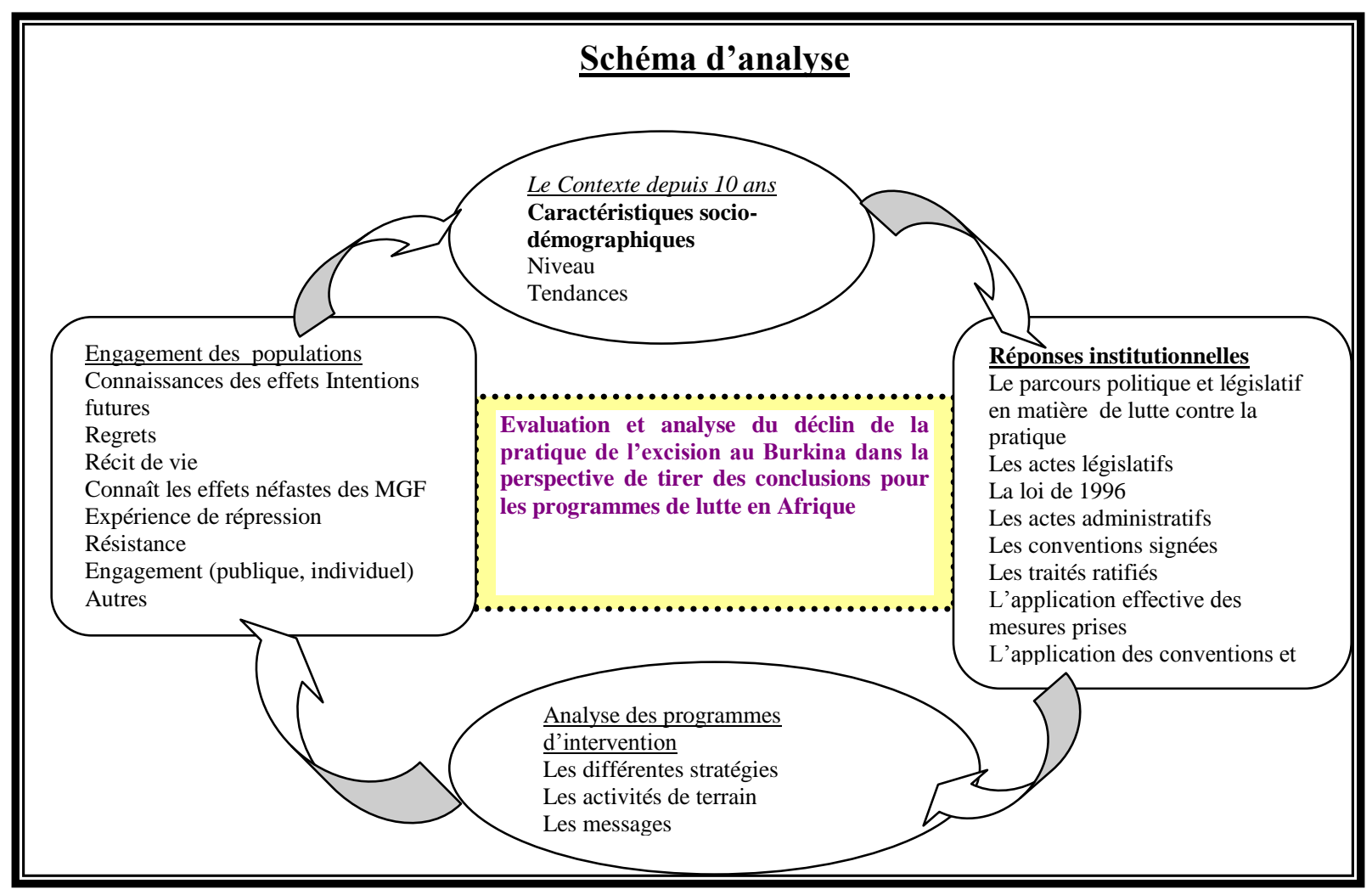

